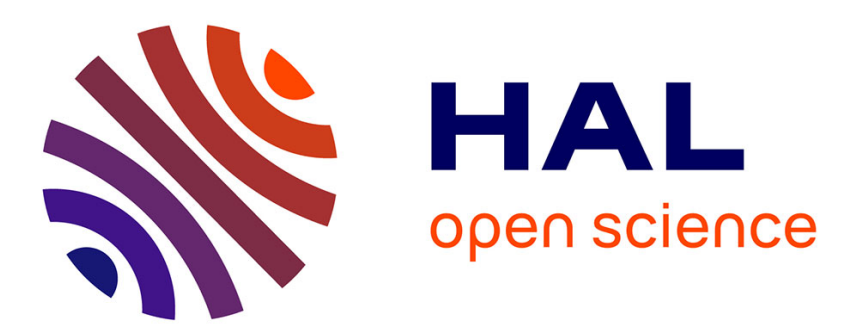

\title{
Robust Winner Determination in Positional Scoring Rules with Uncertain Weights
}

Paolo Viappiani

\section{To cite this version:}

Paolo Viappiani. Robust Winner Determination in Positional Scoring Rules with Uncertain Weights. Theory and Decision, 2020, 88 (3), pp.323-367. 10.1007/s11238-019-09734-3 . hal-02373399

\section{HAL Id: hal-02373399 https://hal.sorbonne-universite.fr/hal-02373399}

Submitted on 20 Nov 2019

HAL is a multi-disciplinary open access archive for the deposit and dissemination of scientific research documents, whether they are published or not. The documents may come from teaching and research institutions in France or abroad, or from public or private research centers.
L'archive ouverte pluridisciplinaire HAL, est destinée au dépôt et à la diffusion de documents scientifiques de niveau recherche, publiés ou non, émanant des établissements d'enseignement et de recherche français ou étrangers, des laboratoires publics ou privés. 
Noname manuscript No.

(will be inserted by the editor)

\title{
Robust Winner Determination in Positional Scoring Rules with Uncertain Weights
}

\author{
Paolo Viappiani
}

the date of receipt and acceptance should be inserted later

\begin{abstract}
Scoring rules constitute a particularly popular technique for aggregating a set of rankings. However, setting the weights associated to rank positions is a crucial task, as different instantiations of the weights can often lead to different winners. In this work we adopt minimax regret as a robust criterion for determining the winner in the presence of uncertainty over the weights. Focusing on two general settings (non-increasing weights and convex sequences of non-increasing weights) we provide a characterization of the minimax regret rule in terms of cumulative ranks, allowing a quick computation of the winner. We then analyze the properties of using minimax regret as a social choice function. Finally we provide some test cases of rank aggregation using the proposed method.
\end{abstract}

Keywords Scoring rules · rank aggregation · computational social choice $\cdot$ possible winners · minimax regret $\cdot$ convex sequences $\cdot$ robust optimization

\section{Introduction}

Rank aggregation arises in many settings including voting, recommender systems, information retrieval and sports. Positional scoring rules are often used in these settings: each alternative receives points based on its position on each of the input rankings and the alternative with the highest total score is deemed the winner.

Scoring rules offer the advantage of being very simple to implement. Moreover, scoring rules satisfy several desirable properties (Young 1975) including monotonicity, consistency and participation (two important properties that are not satisfied by scoring rules are Condorcet consistency and clone-proofness).

In scoring rules, a vector of weights, called scoring vector, determines the amount of points attributed to the first rank, to the second rank, etc. These weights, typically monotone decreasing, can encode preferences for controlling the tradeoff between "extreme" alternatives (often in the first positions and in the last positions), and "moderate" ones (alternatives that are most of the times in the middle positions). A particular scoring rule is Borda count that uses linear weights; while it satisfies some additional properties (Young 1974; Fishburn and Gehrlein 1976) and sometimes presented as superior to other scoring rules, Borda may not be adequate in decision contexts where greater discrimination between the positions is needed. For instance, in many contexts, in particular in sport competitions, it is often assumed that weights should constitute a convex sequence (Llamazares 2016), meaning that the difference between the weight of the first position

Paolo Viappiani

Sorbonne Université

UMR7606 CNRS

LIP6

4 pl. Jussieu, 75005 Paris

France

E-mail: paolo.viappiani@lip6.fr 
and that of the second position is at least as large as the difference between the weight of the second and the third position, and so on.

Setting the weights of a scoring rule is a critical task; indeed, it is often the case that different winners may emerge when using different scoring vectors. Since fixing the scoring vector can be seen as arbitrary choice, Cook and Kress (1990) suggested evaluating each alternative according to its most favorable scoring vector, in order to avoid any subjectivity. Several authors (Cook and Kress 1990; Green et al. 1996; Hashimoto 1997; Foroughi and Tamiz 2005; Llamazares and Peña 2009, 2013; Khodabakhshi and Aryavash 2015; Llamazares 2016) have then proposed similar approaches to score alternatives by considering the space of feasible weights.

In this work we view the rank aggregation problem as a decision problem under uncertainty. The uncertainty over the scoring rule is based on an explicit representation of the possible scoring vectors; this is a setting of strict uncertainty (French 1986). There are several possible criteria that can be used for decision-making under strict uncertainty; the most common are: maximax, maximin, Hurwitz's and minimax regret; the reader is referred to French (1986) for a critical review. In fact, some of previous approaches to rank aggregation can be seen from this prespective: Cook and Kress (1990) adopt the maximax criterion while the criterion chosen by Khodabakhshi and Aryavash (2015) is closely related to Hurwicz's criterion.

While there is no definite consensus on which criterion is best, we think that evaluating each alternative according to its most favorable scoring vector, as done in several of the papers cited above, is often too much "optimistic". As an alternative may perform very well with some scoring vectors, but may have dismal performance according to other scoring vectors, we think that is important to provide some form of robustness. Therefore we adopt minimax regret (Savage 1954) as a criterion for choosing the alternative to be declared as winner; with this robust criterion, we are able to give guarantees on the quality of the decision.

More precisely, we assume that a central authority postulates some basic requirements about the scoring vector, in particular we focus on two settings: 1) the set of non-increasing weights and 2 ) the set of convex non-increasing weights. Each alternative is associated to a max regret value, corresponding to the worst-case loss (in term of score points) that we could incur if we were to choose that alternative. The alternative (or the alternatives) to be declared as "winner" is the one that minimizes such loss, i.e. achieving minimax regret.

In this paper we provide a thorough analysis of winner determination with minimax regret. The main contributions of the paper consist in the characterization of regret in the two main settings (non-increasing weights, non-increasing convex weights), providing closed-form expressions for computing max regret (in Theorem 1 and Theorem 2), in analyzing minimax regret as a social choice function (in Theorem 3), and in providing experimental tests. We also discuss the case in which additional preference information (imposing a minimal value for difference between the value of two weights) is known about the weights.

The paper ${ }^{1}$ is organized as follows. First, in Section 2 we provide relevant background, covering scoring rules with uncertain weights, dominance relations between alternatives, and the identification of possible winners. Then, in Section 3 we present the aggregation with minimax regret and we provide a novel characterization under different hypothesis about the feasible scoring vectors. In Section 4 we present a formal analysis of minimax regret as a social choice function. Finally in Section 5 we describe some tests showing, with real data, the use of the proposed methods for rank aggregation. Section 6 provides some concluding remarks.

\section{Background}

In this section we review relevant background. We begin by presenting the basic decision problem and some notation in Section 2.1. We then reformulate scoring rules in terms of cumulative ranks in Section 2.2. Based on these reformulations, we discuss dominance relations between alternatives in Section 2.3 . Finally, in Section 2.4 we present the notions of possible and necessary winners, and discuss the connection between these concepts and dominance relations.

\footnotetext{
1 Part of this article is based on a conference paper (Viappiani 2018), where we presented some of the theoretical results (without proofs) and discussed the approach based on minimax regret by comparing with expected utility and other criteria. In this article we extend the analysis focusing on minimax regret and study its properties in the context of social choice.
} 


\subsection{Scoring rules}

As usual in social choice, we assume that $n$ voters express preferences in the form of rankings involving a set $A$ of $m$ alternatives (alternatives might be candidates for office, product configurations, restaurant dishes, etc.); rankings are assumed to be linear orders. The set of voters with their preference rankings is known as the profile. Let $v^{x}=\left(v_{1}^{x}, \ldots, v_{m}^{x}\right)$ be the rank distribution of $x$, where $v_{j}^{x}$ is the number of times that alternative $x$ was ranked in the $j$-th position. Note that $\sum_{j=1}^{m} v_{j}^{x}=n$ for each $x \in A$ and $\sum_{x \in A} v_{j}^{x}=n$ for each $j=1, \ldots, m$.

Positional scoring rules discriminate between alternatives by fixing a weight to each rank; the score of an alternative is obtained by summing up the points obtained in each of the input rankings. The score obtained by alternative $x$ is $s(x ; w)=\sum_{j=1}^{m} w_{j} v_{j}^{x}$ where the vector $w=\left(w_{1}, \ldots, w_{m}\right)$, called the scoring vector, specifies the number of points assigned to each position. These total scores can be used to pick a winner or to rank the alternatives from the best to the worst. For example plurality is obtained by setting $w_{1}=1$ and $w_{j}=0$ for all $j \in\{2, \ldots, m\}$.

By choosing a particular $w$, it is possible to specify some preferences on the aggregation, by giving more or less weight to one position compared to the positions that came afterwards in the ranking. We assume that not all weights are null, otherwise the alternatives are not discriminated (degenerated scoring rule). A natural hypothesis is to require that the sequence of weights is nonincreasing: $w_{i} \geq w_{i+1}$ for all $i \in\{1, \ldots, m-1\}$; this expresses the basic intuition that in a ranking an alternative is at least as preferred to the alternatives that comes afterwards.

A scoring rule is invariant to affine positive transformation of the scoring vector. Therefore, with no loss of generality, we let $w_{1}=1$ and $w_{m}=0$ (therefore we have $m-2$ degrees of freedom). Given this assumption the Borda rule is given by setting $w_{j}=\frac{m-j}{m-1}$.

Moreover, it is often (but not always) assumed that the positional weights constitute a convex sequence, meaning that the difference between the first and the second weight is not less than the difference between the second and of the third, and so on. In such a case the weights need to satisfy the following constraint, for each $i$ between 1 and $m-2$ :

$$
w_{i}-w_{i+1} \geq w_{i+1}-w_{i+2} \Longleftrightarrow w_{i}-2 w_{i+1}+w_{i+2} \geq 0
$$

Note that Borda and plurality use a convex scoring vector; furthermore convexity is often satisfied by the weights used when combining ranks in sports, races and other situations (e.g. formula one world championship, alpine skiing world cup).

We now introduce some of the notation that we will use in the paper. We use $\llbracket m \rrbracket$ to denote the set $\{1, \ldots, m\}$. Given two vectors $\alpha$ and $\beta$ of size $m$, we write $\alpha \succeq \beta$ iff $\alpha_{j} \geq \beta_{j}$ for all components $j \in \llbracket m \rrbracket$. If the inequalities are strict, $\alpha_{j}>\beta_{j}$ for all components $j \in \llbracket m \rrbracket$, then we write $\alpha \succ \beta$.

We will use $W^{D}$ to denote the set of scoring vectors with non-increasing weights

$$
W^{D}=\left\{\left(w_{1}, \ldots, w_{m}\right) \mid 1=w_{1} \geq w_{2} \geq \ldots \geq w_{m-1} \geq w_{m}=0\right\},
$$

and $W^{C}$ to denote the set of non-increasing scoring vectors whose weights constitute a convex sequence $^{2}$ (with our boundary assumptions, $w_{1}=1$ and $w_{m}=0$ )

$$
W^{C}=\left\{\left(w_{1}, \ldots, w_{m}\right) \mid w \in W^{D} \wedge w_{i}-2 w_{i+1}+w_{i+2} \geq 0 \quad \forall i \in \llbracket m-2 \rrbracket\right\} .
$$

In order to simplify our terminology, with a little abuse, from now on "decreasing weights" will always mean the set $W^{D}$ of non-increasing weights, and "convex weights" will always refer to $W^{C}$, the set of non-increasing convex weights.

\subsection{Reformulation using Cumulative Ranks}

We now show how it is possible to reformulate scoring rules in terms of cumulative ranks; this is useful in order to establish dominance relations between alternatives in the context of scoring

\footnotetext{
2 There is some redundancy in the constraints: it is enough to assume convexity and $w_{m-1} \geq 0$ to ensure that the sequence is non-increasing.
} 

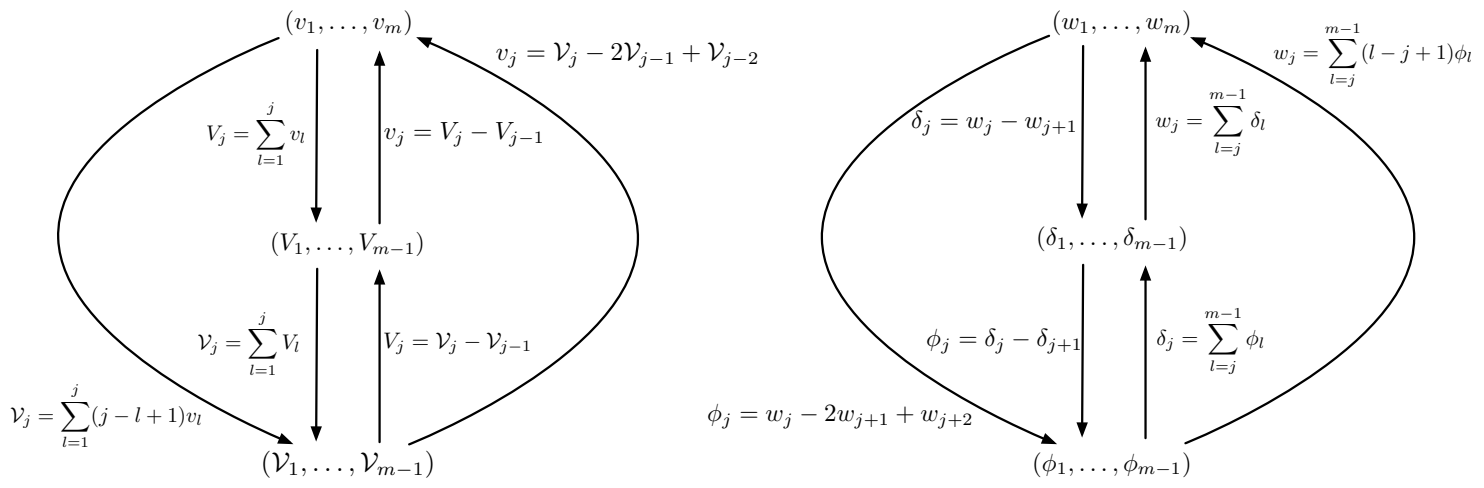

Fig. 1 Relations between rank distribution, cumulative and double cumulative rank distributions (left), between the weights of the scoring vector, differential weights and second-order differential weights (right).

rules, as it will be shown in Section 2.3. The reformulations presented here will also be used in Section 3 to characterize the aggregation method based on minimax regret.

We consider the fraction of times that an alternative was ranked in a certain position or better. Cumulative standings, or cumulative ranks, are defined as the cumulative sum of the rank vectors, starting from the first position. For each $x \in A$, the vector $V^{x}=\left(V_{1}^{x}, \ldots, V_{m-1}^{x}\right)$ is such that $V_{j}^{x}=\sum_{l=1}^{j} v_{l}^{x}$ is the number of times that alternative $x$ has been ranked in position $j$ or better; note that $V^{x}$ has only $m-1$ components (we do not consider the number of times that an alternative was ranked at least in the last place, as it is always equal to the number of voters $n$ ). Cumulative ranks have been previously used by several authors (Stein et al. 1994; Green et al. 1996; Llamazares and Peña 2009, 2015a,b).

We now define the vector $\delta=\left(\delta_{1}, \ldots, \delta_{m-1}\right)$, dubbed differential weights, as the vector of the differences between two successive positional weights of the original scoring vector.

Definition 1 Given the vector of weights $\left(w_{1}, \ldots, w_{m}\right) \in W^{D}$, the vector of differential weights $\delta=\left(\delta_{1}, \ldots, \delta_{m-1}\right)$ is such that

$$
\delta_{j}=w_{j}-w_{j+1} \quad \forall j \in \llbracket m-1 \rrbracket .
$$

Remember that we assumed that $w_{1}=1$ and $w_{m}=0$; this assumption means that we have $\delta_{1}=1-w_{2}$ and $\delta_{m-1}=w_{m-1}$. As in Llamazares and Peña $(2015 \mathrm{a}, \mathrm{b})$, the score obtained by an alternative $x$ can now be expressed as:

$$
s(x ; w)=\sum_{j=1}^{m-1} w_{j} v_{j}^{x}=\sum_{j=1}^{m-1} \sum_{i=j}^{m-1}\left(w_{i}-w_{i+1}\right) v_{j}^{x}=\sum_{j=1}^{m-1} v_{j}^{x} \sum_{i=j}^{m-1} \delta_{i}=\sum_{i=1}^{m-1} \delta_{i} \sum_{j=1}^{i} v_{j}^{x}=\sum_{j=1}^{m-1} \delta_{j} V_{j}^{x} .
$$

This means that the score can be expressed with $\delta$ as parameter, using the cumulative rank $V^{x}$ of $x$. Decreasing weights in the original formulation correspond to positive differential weights; the hypothesis that $w_{1}=1$ implies $\sum_{j=1}^{m-1} \delta_{j}=1$ and therefore the score of an alternative is a convex combination of its vector of cumulative ranks.

Note that the original rank vectors can be expressed in terms of cumulative standings: $v_{j}=V_{j}-$ $V_{j-1}$; similarly the original weights can be recovered from the differential weights: $w_{j}=\sum_{l=j}^{m-1} \delta_{l}$.

We now derive a reformulation that is useful for scoring vectors that constitute convex sequences. To do so we need to introduce the double cumulative rank distribution, also called cumulative of the cumulative standings (Stein et al. 1994; Llamazares 2016):

$$
\mathcal{V}_{j}^{x}=\sum_{l=1}^{j} V_{l}^{x}=\sum_{l=1}^{j} \sum_{o=1}^{l} v_{o}^{x}=\sum_{l=1}^{j}(j-l+1) v_{l}^{x} \quad \forall j \in \llbracket m \rrbracket
$$

We now define a new vector of parameters $\phi$, called second-order differential weights, whose elements represent a discrete analogous of the second-order derivative. 


\begin{tabular}{|c|c|c|c|}
\hline rule & positional weights & differential weights & 2nd-order differential weights \\
\hline Plurality & $w=(1,0, \ldots, 0)$ & $\delta=(1,0, \ldots, 0)$ & $\phi=(1,0, \ldots, 0)$ \\
\hline$k$-approval & $w=(\underbrace{1, \ldots, 1}, 0, \ldots, 0)$ & $\delta=(\underbrace{0, \ldots, 0,1}_{m-2}, 0, \ldots, 0)$ & $\phi=(\underbrace{0, \ldots, 0},-1,1,0, \ldots, 0)$ \\
\hline & $m-k$ & $m-k-1$ & $m-k-1$ \\
\hline Borda & $w=\left(1, \frac{m-2}{m-1}, \frac{m-3}{m-1}, \ldots, \frac{1}{m-1}, 0\right)$ & $\delta=\left(\frac{1}{m-1}, \ldots, \frac{1}{m-1}\right)$ & $\phi=(\underbrace{0, \ldots, 0}_{m-2}, \frac{1}{m-1})$ \\
\hline top-k Borda & $w=(1, \frac{k-1}{k}, \ldots, \frac{1}{k}, \underbrace{0, \ldots, 0}_{m-k})$ & $\delta=(\underbrace{\frac{1}{k}, \ldots, \frac{1}{k}}_{k}, \underbrace{0, \ldots, 0}_{m-k-1})$ & $\phi=(\underbrace{0, \ldots, 0}_{k-1}, \frac{1}{k}, \underbrace{0, \ldots, 0}_{m-k-1})$ \\
\hline
\end{tabular}

Table 1 Common positional scoring rules and their associated (positional, differential, 2nd-order differential) weights.

Definition 2 Given scoring vector $\left(w_{1}, \ldots, w_{m}\right) \in W^{D}$ and the associated differential weights $\left(\delta_{1}, \ldots, \delta_{m-1}\right)$ from Definition 1 , let $\phi$, second-order differential weights, to be the vector $\left(\phi_{1}, \ldots, \phi_{m-1}\right)$ such that

$$
\begin{cases}\phi_{j}=\delta_{j}-\delta_{j+1} & =w_{j}-2 w_{j+1}+w_{j+2} \quad \forall j \in \llbracket m-2 \rrbracket \\ \phi_{m-1}=\delta_{m-1} & =w_{m-1} .\end{cases}
$$

Given that we assumed $w_{1}=1$, we have $\phi_{1}=1-2 w_{2}+w_{3}$ and, since $w_{m}=0$, we have $\phi_{m-2}=$ $\delta_{m-2}-\delta_{m-1}=w_{m-2}-2 w_{m-1}$. Stating $\phi_{j} \geq 0$ for all components $j$ is equivalent to require the scoring vector $w$ to be convex.

The score $s(x ; w)$ of $x$ under a scoring rule with scoring vector $w$ can now be expressed in function of $\phi$,

$$
s(x ; w)=\sum_{j=1}^{m-1} \phi_{j} \mathcal{V}_{j}^{x}
$$

with $\phi$ obtained from $w$ using Equation (3). The constraint that the weights of the scoring vector should be bounded and the highest weight, $w_{1}$ has value equal to one, becomes, expressed in terms of second-order differential weights, the constraint $\sum_{l=1}^{m-1} l \phi_{l}=1$.

In Figure 1 we summarize the relations between rank distributions, cumulative rank distributions and double cumulative distributions, and as well weights, differential and second-order differential (convex) weights. We note that there is a kind of duality between $(v, V, \mathcal{V})$ and $(w, \delta, \phi)$. The scoring vector $w$ is made of non-negative elements, $V$ is non-decreasing and $\mathcal{V}$ is non-decreasing and convex. On the other hand, $w$ is non-increasing and $\delta$ is positive. Furthermore, if $w$ is convex, $\delta$ is non-increasing and $\phi$ is made of non-negative elements.

Example 1 Suppose, for instance, that there are $m=6$ alternatives and the scoring vector is $w=(1,0.6,0.2,0.1,0,0)$. The differential weights are $\delta=(0.4,0.4,0.1,0.1,0)$ and the secondorder differential weights $\phi=(0,0.3,0,0.1,0)$. Note that $w$ is a non-increasing convex sequence (and with 1 and 0 at the extremes); consequently $\delta$ is positive, non-increasing and sums up to 1 ; $\phi$ has all components that are non-negative and is such that $\phi_{1}+2 \phi_{2}+3 \phi_{3}+4 \phi_{4}+5 \phi_{5}=1$. In addition to the usual way, the score of alternative $x$ under $w$ can be computed, using the cumulative distribution (Equation 2), as $0.4 V_{1}^{x}+0.4 V_{2}^{x}+0.1 V_{3}^{x}+0.1 V_{4}^{x}$ or equivalently (using Equation 4) as $0.3 \mathcal{V}_{2}+0.1 \mathcal{V}_{4}$. Assume that the rank distribution of an alternative $x$ is $v^{x}=(3,1,2,0,2,2)$; then the vector of cumulative ranks is $V^{x}=(3,4,6,6,8)$ and the vector of double cumulative ranks is $\mathcal{V}^{x}=(3,7,13,19,27)$. The score $s(x)=\sum_{j=1}^{m-1} \delta_{j} V_{j}^{x}=0.4 \cdot 3+0.4 \cdot 4+0.1 \cdot 6+0.1 \cdot 6=4$ and as well $s(x)=\sum_{j=1}^{m-1} \phi_{j} \mathcal{V}_{j}^{x}=0.3 \cdot 7+0.1 \cdot 19=4$.

As an example of non convex sequence, consider $w^{\prime}=(1,0.2,0.1,0.1,0,0)$ : we have $\delta^{\prime}=$ $(0.8,0.1,0,0.1,0)$ and $\phi^{\prime}=(0.7,0.1,-0.1,0.1,0)$; note that $\delta^{\prime}$ is not monotone $\left(a s \delta_{3}^{\prime}<\delta_{4}^{\prime}\right)$ and $\phi^{\prime}$ is not positive (as $\left.\phi_{3}^{\prime}<0\right)$, indeed the scoring vector $w^{\prime}$ is not convex.

We summarize in Table 1 how some common scoring rules are expressed in terms of differential weights and in terms of second-order differential weights. We highlight the following observations: 
- $k$-approval, the rule obtained with scoring vector $(\underbrace{1, \ldots, 1}_{k}, 0, \ldots, 0)$, is not convex when $k \geq 2$. The $k$-approval score of alternative $x$, denoted as $s_{k \text {-approval }}(x)$, is exactly $V_{k}^{x}$.

- We call top-k Borda the scoring rule based on Borda restricted to giving points to the top $k$ positions. The score $s_{\text {top-k-Borda }}(x)$ of an alternative $x$ with respect to top-k Borda is $\frac{\mathcal{V}_{k}^{x}}{k}$.

\subsection{Dominance relations}

The usefulness of the reformulations presented in Section 2.2 is that they can be used to discriminate alternatives according to dominance relations that allow to identify alternatives that are less preferred than another one for any feasible scoring vector. Dominance only gives us a partial order, so it is usually not enough to unambiguously define a winner. The dominance relations presented here were first discussed by Stein et al. (1994) and can be seen as a special case of those studied by Salo (1995). Note that dominance relations have been widely studied in the context of multi attribute decision problems (Hazen 1986; Weber 1987; Pearman 1993).

Definition 3 Let $x, y \in A$ be two alternatives and let $W$ be a set of scoring vectors.

- Alternative $x$ is said to weakly dominate alternative $y$ with respect to $W$ if the score of $x$ is at least as high as the score of $y$ for any scoring rule with scoring vector in $W$.

- Similarly, alternative $x$ is said to strongly dominate $y$ with respect to $W$ if the score of $x$ is strictly higher than the score of $y$ for any scoring rule with scoring vector in $W$.

- An alternative $x$ is said to be weakly (respectively strongly) dominated if there is another alternative $y$, different from $x$, that weakly (resp. strongly) dominates it.

- An alternative $x$ is said to be weakly undominated if $x$ is not strongly dominated; while alternative $x$ is strongly undominated if $x$ is not weakly dominated.

We now address dominance when considering $W^{D}$ and $W^{C}$ as set of feasible scoring vectors.

Decreasing weights When dealing with scoring vectors in $W^{D}$, i.e. with decreasing weights, the set of possible scores obtained by an alternative $x$, with cumulative ranks $V^{x}$, is given by

$$
\left\{\sum_{j=1}^{m-1} \delta_{j} V_{j}^{x} \mid \delta_{1} \geq 0, \ldots, \delta_{m-1} \geq 0 \wedge \sum_{j=1}^{m-1} \delta_{j}=1\right\} .
$$

Basically, all convex combinations of the components of $V^{x}$ are possible. The next proposition shows that we can compare the cumulative ranks of two alternatives componentwise to check if a dominance relation exists.

Proposition 1 (Stein et al. 1994, Theorem 1, part a) Let $x, y \in A$. Then $x$ weakly dominates $y$ with respect to $W^{D}$ if and only if $V^{x} \succeq V^{y}$, and $x$ strongly dominates $y$ with respect to $W^{D}$ if and only if $V^{x} \succ V^{y}$. In formulas:

$-V^{x} \succeq V^{y} \Longleftrightarrow \forall w \in W^{D} \quad s(x ; w) \geq s(y ; w)$,

$-V^{x} \succ V^{y} \Longleftrightarrow \forall w \in W^{D} \quad s(x ; w)>s(y ; w)$.

The previous statement can be seen as a form of first-order stochastic dominance (Fishburn and Vickson 1978).

Convex weights Assuming a decreasing convex sequence (the scoring vector belongs to $W^{C}$ ), the space of possible scores associated with an alternative $x$ is given by

$$
\left\{\sum_{j=1}^{m-1} \phi_{j} \mathcal{V}_{j}^{x} \mid \phi_{1} \geq 0, \ldots, \phi_{m-1} \geq 0 \wedge \sum_{j=1}^{m-1} j \phi_{j}=1\right\}
$$

All $\phi_{j}$ are non-negative since the scoring vector is assumed to be a convex sequence. 
The next proposition shows that, in the case of convex weights $\left(W^{C}\right)$, we can compare the double cumulative ranks of two alternatives componentwise to check if a dominance relation exists. Indeed, if each element of the vector $\mathcal{V}^{x}$ is at least as big as the corresponding element of $\mathcal{V}^{y}$, then $x$ has at least the same score of $y$ for any scoring rule with convex weights (and the analogous relation holds with strict inequalities).

Proposition 2 (Stein et al. 1994, Theorem 1, part b) Let $x, y \in A$. Then $x$ weakly dominates $y$ with respect to $W^{C}$ if and only if $\mathcal{V}^{x} \succeq \mathcal{V}^{y}$, and $x$ strongly dominates $y$ with respect to $W^{C}$ if and only if $\mathcal{V}^{x} \succ \mathcal{V}^{y}$. In formulas:

$-\mathcal{V}^{x} \succeq \mathcal{V}^{y} \Longleftrightarrow \forall w \in W^{C} \quad s(x ; w) \geq s(y ; w)$,

$-\mathcal{V}^{x} \succ \mathcal{V}^{y} \Longleftrightarrow \forall w \in W^{C} s(x ; w)>s(y ; w)$.

This is akin to second order stochastic dominance (Fishburn and Vickson 1978) in the theory of decision-making under uncertainty, but considering convex and not concave utility.

Example 2 Consider the following numeric example. The first table reports the distribution of the ranks, to be read as follows: a is ranked two times first, two time second, etc., while $b$ is never ranked first but is ranked 6 times second and 2 times third. The second table reports the cumulative ranks (for example, a is ranked 4 times in the second position or better) and the third the double cumulative ranks.

\begin{tabular}{ccccc}
\hline Alternative & $v_{1}$ & $v_{2}^{\cdot}$ & $v_{3}^{\cdot}$ & $v_{4}$ \\
\hline$a$ & 2 & 2 & 2 & 2 \\
$b$ & 0 & 6 & 2 & 0 \\
$c$ & 2 & 0 & 4 & 2 \\
$d$ & 4 & 0 & 0 & 4 \\
\hline
\end{tabular}

\begin{tabular}{cccc}
\hline Alternative & $V_{1}$ & $V_{2}$ & $V_{3}^{\cdot}$ \\
\hline$a$ & 2 & 4 & 6 \\
$b$ & 0 & 6 & 8 \\
$c$ & 2 & 2 & 6 \\
$d$ & 4 & 4 & 4 \\
\hline
\end{tabular}

\begin{tabular}{cccc}
\hline Alternative & $\mathcal{V}_{1}$ & $\mathcal{V}_{2}$ & $\mathcal{V}_{3}$ \\
\hline$a$ & 2 & 6 & 12 \\
$b$ & 0 & 6 & 14 \\
$c$ & 2 & 4 & 10 \\
$d$ & 4 & 8 & 12 \\
\hline
\end{tabular}

- When considering decreasing weights ( set $W^{D}$ ), one can establish dominance by pairwise comparisons of rows in the table of cumulative ranks; for instance alternative $a$ weakly dominates alternative $c$ since $V_{1}^{a}=V_{1}^{c}, V_{2}^{a}>V_{2}^{c}$, and $V_{3}^{a}=V_{3}^{c}$. The set of undominated alternatives is then $\{a, b, d\}$. No strong domination holds.

- When considering convex weights ( set $W^{C}$ ), now alternative $d$ weakly dominates alternative $a$; moreover $d$ strongly dominates $c$ since $\mathcal{V}_{j}^{d}>\mathcal{V}_{j}^{c}$ for all $j \in \llbracket m-1 \rrbracket$. The set of strongly undominated alternatives is $\{b, d\}$, while the set of weakly undominated ones is $\{a, b, d\}$.

\subsection{Possible and necessary winners}

We now introduce the concept of possible and necessary winners of a positional scoring rule with uncertain scoring vector. Note that this differs from the more established notions of possible and necessary winners in voting (Konczak and Lang 2005; Xia and Conitzer 2011), where uncertainty is on the preferences and not on the voting rule.

In what follows, let $W$ be the set of feasible weights; with $W$ being either the set of monotone decreasing weights $W^{D}$ or the set of decreasing convex sequences $W^{C}$.

Definition 4 An alternative $x$ is a possible co-winner, with respect to $W$, if there is a scoring vector $w \in W$ such that the score of $x$ under $w$ is higher or equal than the scores of all other alternatives; that is,

$$
\exists w \in W: s(x ; w) \geq s(y ; w) \forall y \in A \backslash\{x\} .
$$

Moreover, $x$ is said to be a possible winner, with respect to $W$, if there is a scoring vector $w \in W$ such that the score of $x$ under $w$ is strictly higher than the scores of all other alternatives; that is,

$$
\exists w \in W: s(x ; w)>s(y ; w) \forall y \in A \backslash\{x\} .
$$

Definition 5 An alternative $x$ is a necessary co-winner with respect to $W$ if, for all scoring vectors $w \in W$, the score of $x$ under $w$ is higher or equal than the scores of all other alternatives; that is,

$$
\forall w \in W: s(x ; w) \geq s(y ; w) \forall y \in A \backslash\{x\} .
$$




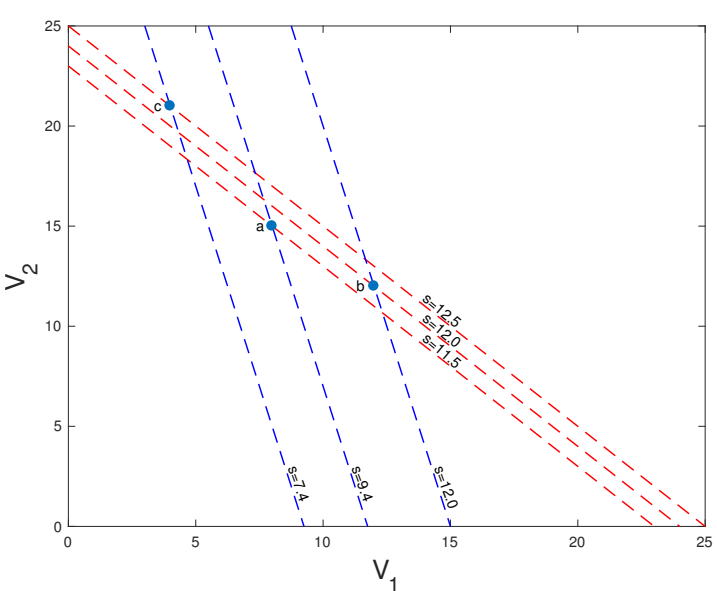

Fig. 2 The indifference curves, points on the $\left(V_{1}, V_{2}\right)$ plane that achieve the same score, are straight lines. We show, as examples, indifference lines for $\delta=(0.5,0.5)$, shown in red, and indifference lines $\delta=(0.8,0.2)$, in blue. There is no way to set $\delta$ in order to make alternative $a$ optimal: either $b$ or $c$ will have higher score than $a$ for any orientation of the indifference lines.

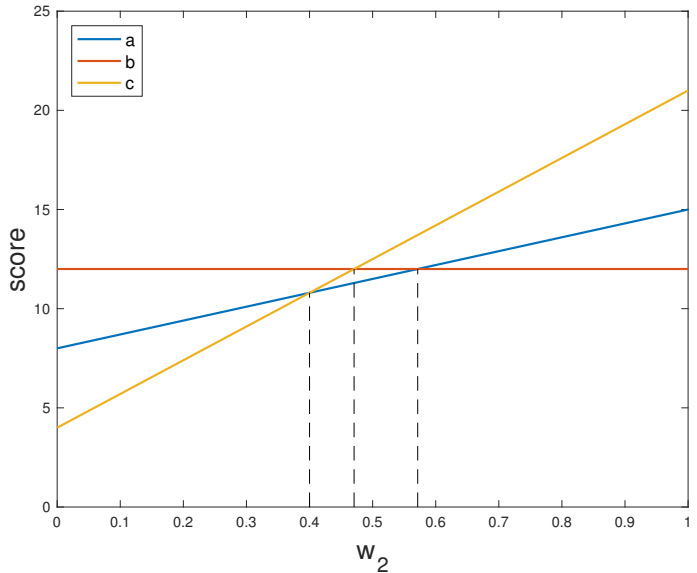

Fig. 3 The scores of $a, b$ in $c$ in function of the parameter $w_{2}$. It can be seen that $a$ cannot be optimal for any value of $w_{2}$, so it is not a possible winner. However it is not dominated by $b$ (there are values of $w_{2}$ that make $a$ better than $b$ ) nor by $c$ (there are values of $w_{2}$ that make $a$ better than $c$ ).

Moreover, $x$ is said to be a necessary winner, with respect to $W$, if, for all scoring vectors $w \in W$, the score of $x$ is strictly higher than the scores of all other alternatives; that is,

$$
\forall w \in W: s(x ; w)>s(y ; w) \forall y \in A \backslash\{x\}
$$

It can be shown that possible co-winners are efficient alternatives in the DEA-AR model of Cook and Kress (1990, page 3).

We now discuss the connection between possible and necessary winners (or co-winners) and the dominance relations described previously in Section 2.3. It is straightforward to realize that a strongly dominated alternative cannot be a possible co-winner, and that a weakly dominated alternative cannot be a possible winner. Moreover the following proposition provides a series of simple observations.

\section{Proposition 3 Let $x \in A$. The following propositions hold:}

- Alternative $x$ is a necessary winner if and only if $x$ strongly dominates every other alternative.

- Alternative $x$ is a necessary co-winner if and only if $x$ weakly dominates every other alternative.

- If there are two or more necessary co-winners, then they all have the same score for all $w \in W$.

- If alternative $x$ is a possible winner, then $x$ is strongly undominated.

- If alternative $x$ is a possible co-winner, then $x$ is weakly undominated.

- If alternative $x$ is a possible co-winner, then either $x$ is strongly undominated or, $x$ is weakly dominated by alternatives that are possible co-winners.

It is important to observe that it is possible to have undominated alternatives that are not possible co-winners (and, therefore, not possible winners). We show this with an example.

Example 3 Assume the rank distributions $(n=24, m=3)$ associated to three alternatives a, $b, c$ presented in the following table (to be read as follows: a is ranked first 8 times, second 7 times and 9 times last).

\begin{tabular}{cccc}
\hline & $v_{1}^{\cdot}$ & $v_{2}^{\cdot}$ & $v_{3}^{\cdot}$ \\
\hline$a$ & 8 & 7 & 9 \\
$b$ & 12 & 0 & 12 \\
$c$ & 4 & 17 & 3 \\
\hline
\end{tabular}


We consider decreasing weights. As usual we let $w_{1}=1$ and $w_{3}=0$, so the only free parameter is $w_{2}$. The score of $a$ is $s(a)=8+7 w_{2}$; the score of $b$ is $s(b)=12$ and the score of $c$ is $s(c)=4+17 w_{2}$. Can a be a winner for some values of $w_{2}$ ? The answer is no: for a to be better than $b$, we need $w_{2} \geq \frac{4}{7}$ but for a to be better than $c, w_{2}$ should be less than $\frac{2}{5}$ (see Figure 2 and Figure 3).

The cumulative ranks are $V^{a}=(8,15), V^{b}=(12,12), V^{c}=(4,21)$ and no pairwise domination holds between $a, b$, or $c$. Therefore $a$ is neither dominated by $b$ or $c$ but is not a possible winner (and not even a possible co-winner).

Note that it is well known in the literature of multi attribute utility theory (Hazen 1986) and multicriteria optimization (Ehrgott 2005) that being undominated does not imply being a possible co-winner. Undominated alternatives are known as Pareto efficient in multicriteria optimization and "supported" solutions are alternatives that maximize a weighted sum of sub-utilities, and the latter are only a subset of the former. The analogy can be fully appreciated by inspecting Equation (2) and (4) to realize that possible co-winners maximize a weighted sum of cumulative ranks.

It is possible to test whether alternative $x$ is a possible winner by computing its maximum advantage, that is the maximum difference between its score and that of the best ranked alternative other than $x$, by considering all possible scoring vectors. If the value of the maximum advantage is positive, then $x$ is a possible winner, while if it is zero then $x$ is a possible co-winner; finally, if the value is negative, there is always at least another alternative that achieves a strictly better score.

Definition 6 The maximum advantage (MA) of an alternative $x \in A$ with respect to $W$ is:

$$
\begin{aligned}
\operatorname{MA}(x ; W) & =\max _{w \in W} \min _{y \in A \backslash\{x\}}\{s(x ; w)-s(y ; w)\}=\max _{w \in W} \min _{y \in A \backslash\{x\}}\left\{\sum_{j=1}^{m} w_{j} v_{j}^{x}-\sum_{j=1}^{m} w_{j} v_{j}^{y}\right\} \\
& =\max _{w \in W}\left\{\sum_{j=1}^{m} w_{j} v_{j}^{x}-\max _{y \neq x} \sum_{j=1}^{m} w_{j} v_{j}^{y}\right\} .
\end{aligned}
$$

The previous definition is general; however, we focus, in this paper, on either the class of decreasing weights or the class of convex weights. The value of MA can be computed with the following optimization:

$$
\begin{array}{ll}
\max _{Z, w} & Z \\
\text { s.t. } & Z \leq \sum_{j=1}^{m}\left(v_{j}^{x}-v_{j}^{y}\right) w_{j} \quad \forall y \in A-\{x\} \\
& w_{j}-w_{j+1} \geq 0 \\
& w_{j}-2 w_{j+1}+w_{j+2} \geq 0 \quad \forall j \in \llbracket m-2 \rrbracket \\
& w_{1}=1 ; w_{m}=0
\end{array}
$$

Equations 7-11 represent a linear program that can be solved with standard optimization tools such as CPLEX or Gurobi. ${ }^{3}$ There are $m-1$ decision variables, of which $m-2$ represent the scoring vector (we have $m-2$ degrees of freedom, since we assume $w_{1}=1$ and $w_{m}=0$ ), and an additional decision variable $Z$ (representing the margin) whose value is constrained (Equation 8) to be less than the difference in score between the score of $x$ and any other alternative $y \in A$. The value at optimum of the decision variable $Z$ gives us the maximum margin $\mathrm{MA}(x ; W)$ of $x$ when choosing $w$ in the set $W$ of feasible scoring vectors. Constraint (10) refers to convex weights (i.e. $W^{C}$ ) and should be removed when dealing with decreasing weights $\left(W^{D}\right)$.

Example 2 (continued) Consider again the values of Example 2, whose distribution of the ranks is given again below. Let us consider, for instance, the computation of the maximum advantage of $b$; the advantage of $b$ with respect to $a$ as a function of $w$ is given by $\sum_{j=1}^{m} w_{j}\left(v_{j}^{b}-v_{j}^{a}\right)=$ $-2 w_{1}+4 w_{2}-2 w_{4}$. The variable $Z$ of the linear program is forced to be simultaneously lower than

\footnotetext{
3 http://www.gurobi.com/
} 
the expressions of the advantage against $a, c$ and $d$; the constraint of Equation 8 above therefore expands to the following constraints:

$$
\begin{aligned}
& Z \leq-2 w_{1}+4 w_{2}-2 w_{4} \\
& Z \leq-2 w_{1}+6 w_{2}-2 w_{3}-2 w_{4} \\
& Z \leq-4 w_{1}+6 w_{2}+2 w_{3}-4 w_{4} .
\end{aligned}
$$

When the problem is solved, the variable $Z$ of the optimum of the linear program gives the value of the maximum advantage. We now give the values of MA obtained by the different alternatives of the example (in the last two column of the table below), once computed w.r.t. $W^{D}$ and once w.r.t. $W^{C}$.

\begin{tabular}{lcccccc}
\hline Alternative & $v_{1}$ & $v_{2}$ & $v_{3}$ & $v_{4}$ & $\mathrm{MA}\left(\cdot ; W^{D}\right)$ & $\mathrm{MA}\left(\cdot ; W^{C}\right)$ \\
\hline$a$ & 2 & 2 & 2 & 2 & 0 & -0.29 \\
$b$ & 0 & 6 & 2 & 0 & 2 & 0.66 \\
$c$ & 2 & 0 & 4 & 2 & 0 & -0.86 \\
$d$ & 4 & 0 & 0 & 4 & 2 & 2 \\
\hline
\end{tabular}

Note that when the weights are assumed to be convex, the feasibility region is reduced and therefore the maximum margin will be lower. Alternatives $b, d$ are possible winners in both cases, since they are associated with a positive maximum advantage. Note that even if a is undominated in $W^{D}, a$ is not a possible winner; in $W^{D}$ both alternatives a and $c$ are possible co-winners; indeed they are co-winners for the scoring vector $w=(1,0.5,0.5,0)$.

\section{Robust winner determination}

The notions of dominance and possible co-winners (presented before in Section 2.3 and 2.4) can be useful to eliminate alternatives that have no possibility of winning without committing to a specific vector of weights. As in general there can be many possible winners or co-winners, we need a method to determine a single winner. While one possibility could be to elicit the weights, using a process similar to that of utility elicitation (Braziunas and Boutilier 2008) adopted in decision aid tools, it is often necessary to make a choice without the possibility of resolving the uncertainty over the weights.

As we mentioned in Section 1, several methods have been proposed in order to determine a winner in presence of an uncertain scoring vector; a review of some of the proposed methods is given by Llamazares and Peña (2009). In particular, the classic work of Cook and Kress (1990) proposed to take an optimistic approach, scoring each alternative according to the score that it can get in the best case, under a number of constraints.

Our point-of-view is that of framing the problem of winner determination as a problem decisionmaking under strict uncertainty. Given that there is a set of feasible weights (either decreasing weights or convex weights) that is deemed possible, we can derive the set of scores that each alternative could attain. Instead of an optimistic approach, we stress the importance of providing some form of robustness in face of the uncertainty over the weights. Assume that a "true" scoring vector exists but it is unknown to us: by adopting the criterion of minimax regret, we pick as winner the alternative that ensures that the worst-case loss (in terms of score), with respect to the winner according to the true (unknown) scoring vector, is minimum.

This Section is structured as follows: in Section 3.1 we discuss the minimax regret criterion, while in Section 3.2 and 3.3 we present a characterization of minimax regret according to two different assumptions about the weight uncertainty that are commonly made in practice: decreasing weights and convex weights. Finally, in Section 3.4 we address the case where additional preferences, relating the values of the weights of consecutive positions, are given about the weights.

\subsection{Minimax regret}

Minimax regret (Savage 1954) is a decision criterion that has been used for robust optimization under uncertainty (Kouvelis and Yu 1997) and as well in decision-making with uncertain utility 
values (Salo and Hämäläinen 2001; Boutilier et al. 2006). Note as well that minimax regret has been recently proposed (Lu and Boutilier 2011) as a way to aggregate incomplete profiles of preferences with a given (fixed) social choice function.

In this article we adopt minimax regret to identify an alternative (to be declared winner) in face of uncertainty over the values of the scoring vector $w=\left(w_{1}, \ldots, w_{m}\right)$. Assuming that the scoring vector $w$ belongs to a set of feasible weights $W$, we measure the quality of an alternative $x$ given $W$ by considering its max regret, whose value represents the worst-case loss (in terms of score "points") that we might incur if we were to choose such an alternative as a winner. In other words, max regret of $x$ measures how far from optimal $x$ could be in the worst case, considering any $w \in W$. The alternative (or the alternatives, in case of ties) associated with minimax regret is is the alternative that is nearest to optimal in the worst case.

The minimax regret approach to winner determination is based on the following definitions.

Definition 7 Given $x \in A$ and $w \in W, \operatorname{Loss}(x ; w)$ is defined as:

$$
\operatorname{Loss}(x ; w)=\max _{y \in A}\{s(y ; w)\}-s(x ; w)
$$

$\operatorname{Loss}(x ; w)$ is the loss (or actual regret) of choosing $x$ as winner instead of choosing the optimal alternative under scoring vector $w$; its value is the difference between the score of the best alternative and the score obtained by alternative $x$ when using the scoring vector $w$. The loss is non-negative.

Definition 8 The pairwise max regret (PMR) of alternative $x$ with respect to alternative $y$ is defined as:

$$
\operatorname{PMR}(x, y ; W)=\max _{w \in W}\{s(y ; w)-s(x ; w)\}=\max _{w \in W}\left\{\sum_{j=1}^{m-1} w_{j} v_{j}^{y}-\sum_{j=1}^{m-1} w_{j} v_{j}^{x}\right\} .
$$

$\operatorname{PMR}(x, y ; W)$, the pairwise max regret of $x$ relative to $y$ given $W$, is the worst-case loss, considering all scoring vectors in $W$, of choosing alternative $x$ rather than $y$; it is the maximum value of the score difference between $y$ and $x$, for any $w \in W$. Notice that pairwise max regret can be negative.

Definition 9 The max regret (MR) of alternative $x$ is defined as:

$$
\begin{aligned}
\operatorname{MR}(x ; W) & =\max _{w \in W} \operatorname{Loss}(x ; w)=\max _{y \in A} \operatorname{PMR}(x, y ; W) \\
& =\max _{y \in A} \max _{w \in W}\left\{\sum_{j=1}^{m-1} w_{j} v_{j}^{y}-\sum_{j=1}^{m-1} w_{j} v_{j}^{x}\right\} .
\end{aligned}
$$

$\operatorname{MR}(x ; W)$ is the worst-case loss associated with picking alternative $x$ as a winner when $w \in W$. We can view max regret as an adversarial selection of the scoring vector $w$ from $W$ in order to maximize the loss between the chosen alternative $x$ and the best alternative under $w$ (the alternative with highest score using $w$ as scoring vector). It can also be viewed as the maximum of the pairwise max regret values of $x$ relative to all alternatives $y \in A$. Max regret is non-negative.

Definition 10 The minimax regret $(M M R)$ and the set $S^{*}$ of regret-optimal alternatives are defined as follows:

$$
\begin{aligned}
\operatorname{MMR}(W) & =\min _{x \in A} \operatorname{MR}(x ; W) \\
S^{*}(W) & =\arg \min _{x \in A} \operatorname{MR}(x ; W)
\end{aligned}
$$

$\operatorname{MMR}(W)$, the minimax regret associated with $W$, is the minimum among the values of max regret of all alternatives in $A$, and the set $S^{*}(W)$ contains the alternatives that achieve this minimal value: for all $x^{*} \in S^{*}(W), \operatorname{MR}\left(x^{*} ; W\right)=\operatorname{MMR}(W)$. The alternatives in $S^{*}(W)$ are to be considered as "winners" according to the minimax regret criterion. Minimax regret is non-negative.

The previous definitions are valid for any set $W$, although in this paper we focus on either $W^{D}$ (decreasing weights) or $W^{C}$ or (convex weights), as these represent typical situations in which scoring rules are used. 


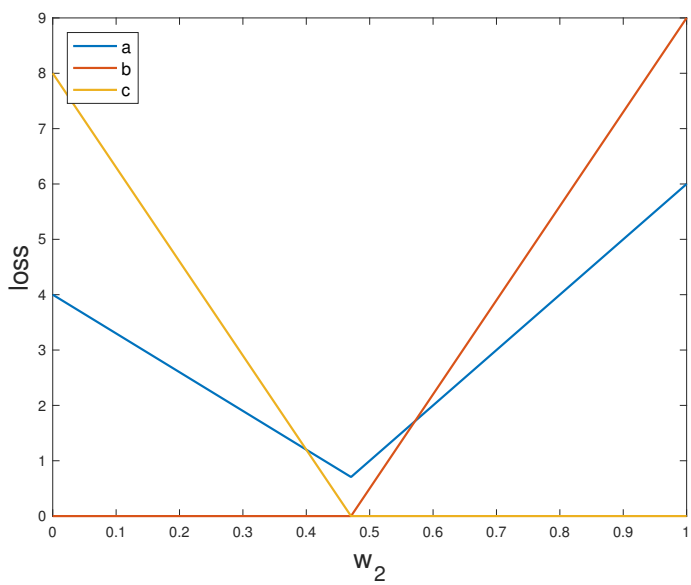

Fig. 4 The loss of $a, b$ in $c$ in function of the parameter $w_{2}$. Max regret is the maximal value of the loss; therefore $\operatorname{MR}(a ; W)=6$, obtained when $w_{2}=1, \operatorname{MR}(b ; W)=9$, obtained when $w_{2}=1$, and $\operatorname{MR}(c ; W)=8$, obtained when $w_{2}=0$; alternative $a$ achieves minimax regret. Note the fact that $a$ always has strictly positive loss: indeed $a$ is not a possible co-winner.

We now provide some general remarks about the use of minimax regret for determining the winner, by inspecting the relation with the notions presented in Section 2: undominated alternatives, possible and necessary winners. First of all, it is immediate to realize from the definition of PMR that, if $x$ weakly dominates $y$ with respect to $W$, then we have $\operatorname{PMR}(x, y ; W) \leq 0$; and similarly, if $x$ strongly dominates $y$, we have $\operatorname{PMR}(x, y ; W)<0$ (and the converse is also true). In the case $\operatorname{PMR}(x, y ; W)=\operatorname{PMR}(y, x ; W)=0$ then $s(x ; w)=s(y ; w)$ for all $w \in W$.

Moreover, ordering the alternatives by their max regret is consistent with dominance in the sense that the relation "having lower max regret" is a refinement of the dominance relations previously introduced, as shown by the next proposition.

Proposition 4 Let $x, y \in A$. The following statements connect max regret and dominance relations:

1. For an arbitrary set $W$ of scoring vectors:

If $x$ weakly dominates $y$, then $\operatorname{MR}(x ; W) \leq \mathrm{MR}(y ; W)$;

if $x$ strongly dominates $y$, then $\operatorname{MR}(x ; W)<\operatorname{MR}(y ; W)$.

2. Moreover, when considering decreasing weights, i.e. the scoring vector belongs to $W^{D}$ : If $V^{x} \succeq V^{y}$ then $\operatorname{MR}\left(x ; W^{D}\right) \leq \operatorname{MR}\left(y ; W^{D}\right)$;

if $V^{x} \succ V^{y}$ then $\operatorname{MR}\left(x ; W^{D}\right)<\operatorname{MR}\left(y ; W^{D}\right)$.

3. Finally, when considering convex weights, i.e. the scoring vector belongs to $W^{C}$ :

If $\mathcal{V}^{x} \succeq \mathcal{V}^{y}$ then $\operatorname{MR}\left(x ; W^{C}\right) \leq \operatorname{MR}\left(y ; W^{C}\right)$;

if $\mathcal{V}^{x} \succ \mathcal{V}^{y}$ then $\operatorname{MR}\left(x ; W^{C}\right)<\operatorname{MR}\left(y ; W^{C}\right)$.

An immediate consequence of the previous proposition is that the alternatives in $S^{*}$, yielding minimax regret, cannot be strongly dominated. An alternative in $x \in S^{*}$ can be weakly dominated however; if this is the case then, according to Proposition $4, x$ is dominated by another alternative in $S^{*}$, and it is undominated with respect to any alternative in $A-S^{*}(W)$.

Proposition 5 The followings statements hold:

1. For any $x \in S^{*}(W), x$ is weakly undominated, i.e. there is no $y \in A$ such that $y$ strongly dominates $x$.

2. For all $x \in S^{*}(W)$, there is no $y \in A \backslash S^{*}(W)$ such that $y$ weakly dominates $x$.

We now turn to inspecting necessary winners and co-winners. Since $\operatorname{MR}(x ; W)$ bounds the loss of choosing $x, \operatorname{MR}(x ; W)=0$ means that $x$ is a necessary co-winner: a max regret value of 0 ensures that no other alternative can be strictly better. Therefore when minimax regret is 0 , the alternatives in $S^{*}$ are necessary co-winners. When an alternative $x$ is a necessary winner, then $\operatorname{MMR}(W)=\operatorname{MR}(x ; W)=0$ and $S^{*}=\{x\}$ (all other alternatives have strictly higher max regret). 
Proposition 6 Let $x \in A$. The following statements hold:

1. Alternative $x$ is a necessary co-winner if and only if $\operatorname{MR}(x ; W)=0$.

2. If $x$ is a necessary winner then $\operatorname{MR}(x ; W)=0$ and $S^{*}(W)=\{x\}$.

3. Alternative $x$ is a necessary winner if and only if $\operatorname{PMR}(x, z ; W)<0$ for all $z \in A \backslash\{x\}$.

We now show that the proposed aggregation method based on minimax regret might pick as a winner an alternative that is not a possible co-winner for any scoring vector.

Observation 1 An alternative associated with minimax regret may not be a possible co-winner.

This is shown with an example.

Example 3 (continued) We consider again the example with 3 alternatives (see page 8) with cumulative scores $V^{a}=(8,15), V^{b}=(12,12)$ and $V^{c}=(4,21)$. We assume that the scoring vector lies in $W^{D}$ (decreasing weights). We have seen before that no pairwise domination hold and that a is not a possible winner.

Figure 4 plots the value of $\operatorname{Loss}\left(x ; W^{D}\right)$ for $x \in\{a, b, c\}$ as a function of $w_{2}$ (the value of the loss of $x$ corresponds, for each $w$, to the difference between the highest score for each $w$ and the score of $x$, and can be found graphically by looking at Figure 3). Inspecting Figure 3, we find that the max regret values are the following: $\operatorname{MR}\left(a ; W^{D}\right)=6, \operatorname{MR}\left(b ; W^{D}\right)=9$ and $\operatorname{MR}\left(c ; W^{D}\right)=8$. Alternative $a$ is the one that guarantees that the loss with respect to the best alternative is lowest. Therefore $a$ is the best alternative according to max regret, although there is no scoring rules with weights in $W^{D}$ that makes it a winner.

The paiwise max regret $\operatorname{PMR}(x, y ; W)$ can be computed using a linear program; the objective function is expressed as

$$
\max \left(v_{1}^{y}-v_{1}^{x}\right)+\sum_{j=2}^{m-1} w_{j}\left(v_{j}^{y}-v_{j}^{x}\right)
$$

where the decision variables are $w_{2}, \ldots, w_{m-1}$. One needs to impose linear constraints on the decision variables in order to force that the scoring vector (the weights) is a feasible instantiation. The constraints $w_{j} \geq w_{j+1}$ for $j \in \llbracket m-2 \rrbracket$ and $w_{m-1} \geq 0$ express the fact that the scoring vector should belong to $W^{D}$. In the case of $W^{C}$ (when a convex sequence is desired) the constraint $w_{j}-2 w_{j+1}+w_{j+2} \geq 0$ has to be added for each $j \in \llbracket m-2 \rrbracket$.

While of course the use of linear programming is a perfectly valid approach, in the following we provide characterizations of minimax regret in terms of cumulative ranks and double cumulative ranks. The theoretical results, that we present next, give us a way to compute max regret without solving an optimization problem; therefore we can compute the winner(s) in a very efficient way. We first address decreasing weights in Section 3.2 and then address convex weights in Section 3.3. Finally, in Section 3.4 we address the case where additional preferences, relating the values of the weights of consecutive positions, are given about the weights.

3.2 Characterization of max regret with decreasing weights

We now analyze the minimax regret criterion in the case that the scoring vector belongs to $W^{D}$. In the following lemma we give a characterization of the pairwise max regret in terms of cumulative ranks.

Lemma 1 In the case of decreasing weights, for any pairs $x, y \in A$, it holds

$$
\operatorname{PMR}\left(x, y ; W^{D}\right)=\max _{j \in \llbracket m-1 \rrbracket}\left\{V_{j}^{y}-V_{j}^{x}\right\} .
$$


Remember that the score obtained with k-approval is $s_{\mathrm{k} \text {-approval }}(x)=s\left(x ; w_{\mathrm{k} \text {-approval }}\right)=V_{k}^{x}$, where $w_{\mathrm{k} \text {-approval }}$ is the vector with 1 in the first $k$ position and 0 afterwards (refer to the end of Section 2.2 and Table 1). Then, according to Lemma 1,

$$
\operatorname{PMR}\left(x, y ; W^{D}\right)=\max _{j \in \llbracket m-1 \rrbracket} s_{\text {j-approval }}(y)-s_{\text {j-approval }}(x)
$$

that is the maximum difference of scores with respect to plurality, 2-approval, 3-approval, etc. Note also that the winner according to $k$-approval is then just the alternative with highest value $V_{k}$; let $V_{k}^{*}=\max _{x \in A} V_{k}^{x}$ be such value.

The following theorem makes use of Lemma 1 and characterize the value of max regret in case of decreasing weights.

Theorem 1 For any alternative $x \in A$ it holds

$$
\operatorname{MR}\left(x ; W^{D}\right)=\max _{j \in \llbracket m-1 \rrbracket}\left\{V_{j}^{*}-V_{j}^{x}\right\}
$$

where $V_{k}^{*}=\max _{x \in A} V_{k}^{x}$.

The importance of Theorem 1 is that it is possible to compute max regret without solving any optimization, considering the maximum difference between cumulative ranks. Note that, since $V_{j}^{*}$ is equivalent to the best score according to $j$-approval, the term $V_{j}^{*}-V_{j}^{x}$ is the loss occurred to $x$ when considering $j$-approval; max regret can be seen as the maximum loss occurred by $x$ with respect to the family of $k$-approval voting rules.

The value of minimax regret is therefore $\operatorname{MMR}(W)=\min _{x \in A} \max _{j \in \llbracket m-1 \rrbracket}\left\{V_{j}^{*}-V_{j}^{x}\right\}$. The minimax regret optimal alternative is then the alternative that is the least far away from the optimal score attained with any $k$-approval voting rule.

Example 2 (continued) We provide an example of max regret computation using Theorem 1, considering again the rank distribution of Example 2. We assume $w \in W^{D}$ (decreasing weights). One needs to consider the table of the cumulative standings; the max regret of a given alternative is the maximum shortfall between the values in the alternative's row compared to the best value (in bold in the tables below) in each column. Hence, $\operatorname{MR}\left(a ; W^{D}\right)=\max \{4-2,6-4,8-6\}=2$.

\begin{tabular}{ccccc}
\hline Alternative & $v_{1}^{*}$ & $v_{2}^{*}$ & $v_{3}^{\cdot}$ & $v_{4}^{*}$ \\
\hline$a$ & 2 & 2 & 2 & 2 \\
$b$ & 0 & 6 & 2 & 0 \\
$c$ & 2 & 0 & 4 & 2 \\
$d$ & 4 & 0 & 0 & 4 \\
\hline
\end{tabular}

\begin{tabular}{cccc}
\hline Alternative & $V_{1}^{*}$ & $V_{2}^{*}$ & $V_{3}^{*}$ \\
\hline$a$ & 2 & 4 & 6 \\
$b$ & 0 & $\mathbf{6}$ & $\mathbf{8}$ \\
$c$ & 2 & 2 & 6 \\
$d$ & $\mathbf{4}$ & 4 & 4 \\
\hline
\end{tabular}

\begin{tabular}{cc}
\hline Alternative & $\mathrm{MR}\left(\cdot ; W^{D}\right)$ \\
\hline$a$ & $\mathbf{2}$ \\
$b$ & 4 \\
$c$ & 4 \\
$d$ & 4 \\
\hline
\end{tabular}

The regret-optimal alternative, that is the alternative with minimum $\operatorname{MR}\left(\cdot ; W^{D}\right)$, is therefore alternative $a$, and $\operatorname{MMR}\left(W^{D}\right)=\operatorname{MR}\left(a ; W^{D}\right)=2$.

\subsection{Characterization of max regret with convex weights}

We continue the analysis by considering convex weights, assuming $w \in W^{C}$. With this assumption, the space of possible scores associated to an alternative is given by

$$
\left\{\sum_{j=1}^{m-1} \phi_{j} \mathcal{V}_{j}^{x} \mid \phi_{1} \geq 0, \ldots, \phi_{m-1} \geq 0 \wedge \sum_{j=1}^{m-1} j \phi_{j}=1\right\}
$$

We now provide a characterization of max regret with respect to $W^{C}$ in terms of double cumulative ranks, therefore allowing to compute the minimax regret optimal alternative very easily. First of all, we consider pairwise max regret.

Lemma 2 Assuming convex weights, the pairwise max regret of an alternative $x$ against $y$ can be computed as follows:

$$
\operatorname{PMR}\left(x, y ; W^{C}\right)=\max _{j \in \llbracket m-1 \rrbracket}\left\{\frac{\mathcal{V}_{j}^{y}-\mathcal{V}_{j}^{x}}{j}\right\} .
$$


Note that $\frac{\mathcal{V}_{k}^{x}}{k}$ represents the score associated to the top-k Borda rule (see the end of Section 2.2 and Table 1$)$. Then, according to Lemma 2 ,

$$
\operatorname{PMR}\left(x, y ; W^{C}\right)=\max _{j \in \llbracket m-1 \rrbracket} s_{\text {top-j-Borda }}(y)-s_{\text {top-j-Borda }}(x) .
$$

Let $\mathcal{V}_{j}^{*}=\max _{x \in A} \mathcal{V}_{j}^{x}$ be the maximum value of the double cumulative ranks for a given position $j$. We use Lemma 2 to derive the following Theorem that provides the characterization of max regret under convex weights.

Theorem 2 Assuming convex weights, the max regret of alternative $x$ can be computed as follows:

$$
\operatorname{MR}\left(x ; W^{C}\right)=\max _{j \in \llbracket m-1 \rrbracket}\left\{\frac{\mathcal{V}_{j}^{*}-\mathcal{V}_{j}^{x}}{j}\right\}
$$

where $\mathcal{V}_{j}^{*}=\max _{x \in A} \mathcal{V}_{j}^{x}$.

Equation 24 basically states that the computation of max regret when $w$ lies in $W^{C}$ is equivalent to consider the maximum loss occurred by $x$ with respect to the family of top- $k$ Borda aggregators (that includes plurality and Borda as a special case). The practical usefulness of Theorem 2 is that it allows to compute the value of max regret of an alternative in closed form (therefore without having to solve a linear program).

Example 2 (continued) We consider again the running example and compute the max regret values when considering that the scoring vector is a convex sequence, using Theorem 2. First, we compute the double cumulative distribution of the ranks; we then divide the second column by two, the third by three, etc. Then, for computing $\operatorname{MR}\left(a ; W^{C}\right)$, we consider the maximum, among columns, between the value of $a$ and the best (bold) value, i.e. $\max \{4-2,4-3,4.66-4\}=2$. The computation is similar for the other alternatives; we obtain that the minimax regret optimal alternative in this case is therefore $d$, yielding $\operatorname{MR}\left(d ; W^{C}\right)=0.66$.

\begin{tabular}{lcccc}
\hline Alternative & $\frac{\mathcal{V}_{1}}{1}$ & $\frac{\mathcal{V}_{2}}{2}$ & $\frac{\mathcal{V}_{3}}{3}$ & $\mathrm{MR}\left(\cdot, W^{C}\right)$ \\
\hline$a$ & 2 & 3 & 4 & 2 \\
$b$ & 0 & 3 & $\mathbf{4 . 6 6}$ & 4 \\
$c$ & 2 & 2 & 3.33 & 2 \\
$d$ & $\mathbf{4}$ & $\mathbf{4}$ & 4 & $\mathbf{0 . 6 6}$ \\
\hline
\end{tabular}

3.4 Computing max regret in presence of additional preferences

In some circumstances it is expected that there is at least some difference between successive weights; as for instance in Cook and Kress (1990). Indeed, in addition of assuming decreasing weights, one might want to impose a minimum difference between consecutive weights and to specify different values for different positions:

$$
w_{j}-w_{j+1} \geq t_{j} \quad \forall j \in \llbracket m-1 \rrbracket
$$

with $t_{1}, \ldots, t_{m-1}$ being non-negative scalars. These thresholds are called discriminative values in the following. We consider:

- $W^{D, t}$, the set of decreasing weights with discriminative values (thresholds) $t_{1}, \ldots, t_{m-1}$, and

- $W^{C, t}$, the set of convex weights with discriminative values (thresholds) $t_{1}, \ldots, t_{m-1}$.

We now analyze how minimax regret can be computed in these two settings. 
Decreasing weights The next proposition provides us with an efficient way to compute pairwise max regret, that is computing the scoring vector $w \in W^{D, t}$ that maximizes the loss $s(y ; w)-s(x ; w)$.

Proposition 7 For any pair of alternatives $x, y \in A$, it holds

$$
\operatorname{PMR}\left(x, y ; W^{D, t}\right)=\left[\left(1-\sum_{j=1}^{m-1} t_{j}\right) \max _{j \in \llbracket m-1 \rrbracket}\left\{V_{j}^{y}-V_{j}^{x}\right\}\right]+\sum_{j=1}^{m-1} t_{j}\left(V_{j}^{y}-V_{j}^{x}\right)
$$

or, equivalently

$$
\operatorname{PMR}\left(x, y ; W^{D, t}\right)=\left[\left(1-\sum_{j=1}^{m-1} t_{j}\right) \operatorname{PMR}\left(x, y ; W^{D}\right)\right]+\sum_{j=1}^{m-1} t_{j}\left(V_{j}^{y}-V_{j}^{x}\right)
$$

where $\operatorname{PMR}\left(x, y ; W^{D}\right)$ is pairwise max regret computed with no discriminative thresholds.

Note that Proposition 7 can as well be used to check dominance with respect to $W^{D, t}$ by testing the sign of $\operatorname{PMR}\left(x, y ; W^{D, t}\right)$.

The previous proposition directly leads to the formulation of max regret, by substituting Equation (26) into Equation (17):

$$
\operatorname{MR}\left(x ; W^{D, t}\right)=\max _{y \in A}\left\{\left(1-\sum_{j=1}^{m-1} t_{j}\right) \max _{j \in \llbracket m-1 \rrbracket}\left\{V_{j}^{y}-V_{j}^{x}\right\}+\sum_{j=1}^{m-1} t_{j}\left(V_{j}^{y}-V_{j}^{x}\right)\right\} .
$$

Convex weights The remaining case to consider is that of $W^{C, t}$, the set of convex weights with discriminative thresholds. For computing the max regret in this case, we do not have a closed formula. Note that the following simple linear program computes the pairwise max regret, that is equal to maximum score difference between $y$ and $x$ when the scoring vector $w$ is convex with discriminative thresholds $t$.

$$
\begin{aligned}
& \max \sum_{j=1}^{m-1} \delta_{j}\left(V_{j}^{y}-V_{j}^{x}\right) \\
& \text { s.t. } \delta_{j} \geq \delta_{j+1} \\
& \delta_{j} \geq t_{j} \\
& \forall j \in \llbracket m-2 \rrbracket \\
& \sum_{j=1}^{m-1} \delta_{j}=1
\end{aligned}
$$

Constraint 28, 29 and 30 assure that the weights are in $W^{C, t}$. When the result of the optimization above is lower than 0 , than $y$ dominates $x$. We use the linear program of Equations 27-30 to compute the pairwise max regret of alternative $x$ against each alternative $y \in A$, and take the maximum value, to find $\operatorname{MR}\left(x ; W^{C, t}\right)$.

Example 2 (continued) We consider again the running example. We compare the scores obtained by evaluating each alternative according to max regret using the following threshold vectors:

$-t^{1}=(0.2,0.1,0.1)$,

$-t^{2}=(0.3,0.1,0.1)$

$-t^{3}=(0.3,0,0)$,

$-t^{4}=(0,0.1,0.4)$.

In the following table we show the scores obtained by the alternatives with max regret with the threshold vectors $t_{1}, \ldots, t_{4}$ (in the first row we show the regret values obtained with no discriminative thresholds) assuming the space $W^{D, t}$. Note that, with different threshold vectors, any of the undominated alternatives $a, b, d$ may be optimal with respect to minimax regret.

$W e$ then consider minimax regret with the scoring vector belonging to the space $W^{C, t}$. We show the scores obtained by using max regret to evaluate the alternatives with the different vectors $t_{1}, t_{2}$ and $t_{3}$ (as $t_{4}$ is not consistent with convexity). In all cases, $d$ achieves minimax regret. 


\begin{tabular}{|c|c|c|c|c|c|c|c|c|}
\hline \multirow{2}{*}{$\begin{array}{l}\mathrm{MR} \\
t\end{array}$} & \multicolumn{4}{|c|}{ Decreasing } & \multicolumn{4}{|c|}{ Convex } \\
\hline & $a$ & $b$ & $c$ & $d$ & $a$ & $b$ & $c$ & $d$ \\
\hline$(0,0,0)$ & 2 & 4 & 4 & 4 & 2 & 4 & 2 & 0.66 \\
\hline$t^{1}$ & 1.4 & 2.6 & 2.6 & 2.2 & 1.4 & 2.6 & 1.6 & 0.66 \\
\hline$t^{2}$ & 1.4 & 2.6 & 2 & 1.4 & 1.4 & 2.6 & 1.6 & 0.66 \\
\hline$t^{3}$ & 2 & 4 & 2 & 0.8 & 2 & 4 & 2 & 0.2 \\
\hline$t^{4}$ & 2 & 0.2 & 3.2 & 3.8 & & & & \\
\hline
\end{tabular}

Example 4 We consider the following rank distribution table, taken from Llamazares and Peña (2013), with 6 alternatives and 10 voters; however it is assumed that only the top 4 positions contribute to the score (and therefore the rank distribution is given only for the first 4 positions).

\begin{tabular}{ccccc}
\hline Alternative & $v_{1}^{*}$ & $v_{2}^{*}$ & $v_{3}^{*}$ & $v_{4}$ \\
\hline$a$ & 3 & 3 & 4 & 3 \\
$b$ & 4 & 5 & 5 & 2 \\
$c$ & 6 & 2 & 3 & 2 \\
$d$ & 6 & 2 & 2 & 6 \\
$e$ & 0 & 4 & 3 & 4 \\
$f$ & 1 & 4 & 3 & 3 \\
\hline
\end{tabular}

We note that alternatives $a, e$ and $f$ are dominated. In the table below we summarize the result of the aggregation with regret for different preferences about the scoring vector. When assuming decreasing weights, max regret ranks $\langle b, c, d\rangle$, but the reverse ranking $\langle d, c, b\rangle$ is obtained when assuming convex weights.

We also show the max regret values obtained with different values of the vector $t$ of discriminative thresholds. Depending on vector $t$, any of the undominated alternatives $b$, $c$ or $d$ may be selected by the aggregation method.

\begin{tabular}{|c|c|c|c|c|c|c|c|c|c|c|c|c|}
\hline \multirow{2}{*}{$\begin{array}{l}\mathrm{MR} \\
t\end{array}$} & \multicolumn{6}{|c|}{ Decreasing } & \multicolumn{6}{|c|}{ Convex } \\
\hline & $a$ & $b$ & $c$ & $d$ & $e$ & $f$ & $a$ & $b$ & $c$ & $d$ & $e$ & $f$ \\
\hline$(0,0,0,0)$ & 4 & 2 & 3 & 4 & 7 & 6 & 3 & 2 & 1.25 & $\mathbf{1}$ & 6 & 5 \\
\hline$(0.2,0.2,0.1,0)$ & 3.20 & 0.90 & 1.60 & 2.20 & 6.00 & 5.00 & 2.75 & 0.90 & 1.25 & 1.00 & 5.40 & 4.50 \\
\hline$(0.4,0,0,0)$ & 3.00 & 2.00 & 1.80 & 1.60 & 6.00 & 5.00 & 3.00 & 2.00 & 0.60 & 0.70 & 6.00 & 5.00 \\
\hline$(0.3,0,0,0.1)$ & 3.00 & 1.80 & 2.10 & 1.80 & 5.90 & 5.00 & 2.70 & 0.90 & 1.03 & 0.90 & 5.40 & 4.50 \\
\hline$(0.44,0,0.16,0)$ & 2.68 & 1.20 & 1.04 & 1.36 & 5.68 & 4.68 & 2.52 & 0.72 & 0.43 & 0.52 & 5.36 & 4.36 \\
\hline
\end{tabular}

\section{Analysis of Aggregation with Minimax Regret as a Social Choice Function}

We now ask ourselves whether the proposed aggregation method satisfies the traditional properties that are studied in the theory of social choice (Zwicker 2016). Remember that a profile is the set of input rankings, and let $P$ be the set of profiles (all possible ways to combine $n$ permutations of $m$ alternatives). A social choice function is a function $f: P \rightarrow 2^{A} \backslash \emptyset$ associating each possible profile with a set of "winners" (a non empty subset of $A$, typically small).

In the following, for the rest of this section, we will view minimax regret as a social choice function, mapping a profile to a set of winners. Note that the information basis is the rank distribution, imposing an equivalence relation between profiles that induces that same rank distribution $v^{x}$ for each alternative $x \in A$. As we need a notation to make explicit the profile we are referring to, we write $v^{x}[p]$ for the rank distribution of $x$ is with respect to profile $p$.

Definition 11 We define the following social choice functions:

- The minimax regret rule with decreasing weights (MMR-Dec) is defined as follows: for any profile $p$, MMR-Dec returns the set $S^{*}\left(W^{D}\right)$ or alternative yielding minimax regret, computed with the rank distribution derived by $p$.

- The minimax regret rule with convex weights (MMR-Con) is defined as follows: for any profile $p$, MMR-Con returns the set $S^{*}\left(W^{C}\right)$ or alternative yielding minimax regret, computed with the rank distribution derived by the profile $p$. 
It is easy to notice that MMR-Dec and MMR-Con are neutral, anonymous (there is no bias in favour of any alternative or voter) and unanimous (if all voters place an alternative in first position, this alternative is selected as winner).

The Pareto property requires that no Pareto dominated alternative is selected as winner or co-winner. Alternative $y$ is said to be Pareto dominated if there is another alternative $x$ such that $x$ is preferred to $y$ by all voters. Bur if in all input rankings $x$ occupies a better rank position than $y$, then it must then be $V^{x} \succeq V^{y}$. This guarantees that the alternatives yielding minimax regret are not strongly dominated; but, unfortunately, weak dominance cannot be excluded (see Proposition 5) and not even Pareto dominance. Therefore the Pareto property is actually not satisfied.

Note however that is straightforward to amend the rules to ensure that Pareto is satisfied: Proposition 5 ensures that weak dominance can only hold among alternatives that are tied at the minimax regret value. Therefore, it is enough to filter out the weakly dominated alternatives (by comparing the cumulative ranks, in the case of $W^{D}$, and by comparing double cumulative ranks, in the case of $W^{C}$ ), if there are any, from the set of alternatives that achieve minimax regret (since Pareto dominated alternatives are also weakly dominated, filtering out weakly dominated alternatives ensure that the remaining ones are Pareto undominated).

Monotonicity requires that, if some voter raises a winning alternative in their ranking without changing the orders of the remaining ones, that alternative continues to be a winner. Aggregation of rankings using minimax regret is monotonic. Intuitively, if $x$ is a winner in a profile $p$, it has to continue to be a winner in a $p^{\prime}$ obtained by placing $x$ in in a better position, since it will be associated with the same value of max regret or lower; we conclude that the rule is monotone.

Our rules do not satisfy Independence to irrelevant alternatives (IIA). The lack of satisfaction of IIA is not however necessarily problematic, since it is often this property that is relaxed in order to escape the Arrow's theorem; moreover it can be argued that decisions in real contexts are often impacted by the set of available decisions ${ }^{4}$.

A weaker form of IIA is to require that the aggregation is independent of the rank of irrelevant alternatives. This property, expressed in terms of social ranking, require that any change in ranks of alternatives $A \backslash\{x, y\}$ should not change the order between $x$ and $y$. In terms of social choice function, we interpret this as requiring that if $x$ is a winner and $y$ is not, any change in ranks of alternatives $A \backslash\{x, y\}$ should not make $y$ winner. While scoring rules and the method proposed by Llamazares and Peña (2013) satisfy this weaker property, minimax regret does not, as shown by the following example.

Example 2 (continued) Let us denote p the original profile of Example 2 and consider the profile $p^{\prime}$, where alternatives $b$ and $c$ have different rank distributions, while the rank distributions of alternatives $a$ and $d$ are unchanged. In $p^{\prime}$ all alternatives are associated with the same max regret value of 3 and in particular now alternatives a and $d$ are considered equal, while in the original example alternative a was the only winner and had a lower max regret value than $d$.

\begin{tabular}{ccccc}
\hline Alternative & $v_{1}$ & $v_{2}$ & $v_{3}$ & $v_{4}$ \\
\hline$a$ & 2 & 2 & 2 & 2 \\
$b$ & 1 & 6 & 0 & 1 \\
$c$ & 1 & 0 & 6 & 1 \\
$d$ & 3 & 0 & 1 & 4 \\
\hline
\end{tabular}

\begin{tabular}{ccccc}
\hline Alternative & $V_{1}^{*}$ & $V_{2}^{*}$ & $V_{3}^{*}$ & $M R$ \\
\hline$a$ & 2 & 4 & 6 & 3 \\
$b$ & 1 & 7 & 7 & 3 \\
$c$ & 1 & 1 & 7 & 3 \\
$d$ & 4 & 4 & 4 & 3 \\
\hline
\end{tabular}

We now considered an even weaker form of IIA that we call Independence from the Rank of Dominated Alternatives (IRDA $)^{5}$. IRDA states that the set of winners does not change whenever a profile is modified in a way that everything stays the same beside the ranks of dominated alternatives.

Definition 12 A social choice function $f$ satisfies Independence from the Rank of Dominated Alternatives (IRDA) if, for any two profiles $p, p^{\prime}$, defined on the same alternatives $A$, we have that $f(p)=f\left(p^{\prime}\right)$, the winners are the same in $p$ and $p^{\prime}$, whenever

\footnotetext{
4 See for instance the discussion on "Minimax and the objection from irrelevant alternatives" in Peterson's book (see Peterson 2017, pag. 53).

5 Indeed Llamazares and Peña Llamazares and Peña (2009) remarks that winner determination should not be sensible to the ranks obtained by "inefficient" alternatives.
} 
Profile 1:

\begin{tabular}{ccccc}
\hline Alternative & $v_{1}^{*}$ & $v_{2}^{*}$ & $v_{3}^{*}$ & $v_{4}$ \\
\hline $\mathrm{a}$ & 4 & 0 & 2 & 0 \\
$\mathrm{~b}$ & 1 & 5 & 0 & 0 \\
$\mathrm{c}$ & 1 & 1 & 4 & 0 \\
$\mathrm{~d}$ & 0 & 0 & 0 & 6
\end{tabular}

\begin{tabular}{ccccc}
\hline Alternative & $V_{1}^{*}$ & $V_{2}^{*}$ & $V_{3}^{*}$ & $\mathrm{MR}$ \\
\hline $\mathrm{a}$ & $\mathbf{4}$ & 4 & $\mathbf{6}$ & 2 \\
$\mathrm{~b}$ & 1 & $\mathbf{6}$ & $\mathbf{6}$ & 3 \\
$\mathrm{c}$ & 1 & 2 & $\mathbf{6}$ & 3 \\
$\mathrm{~d}$ & 0 & 0 & 0 & 6 \\
\hline
\end{tabular}

Profile 2:

\begin{tabular}{ccccc}
\hline \hline Alternative & $v_{1}^{*}$ & $v_{2}^{*}$ & $v_{3}^{*}$ & $v_{4}$ \\
\hline $\mathrm{a}$ & 2 & 0 & 5 & 0 \\
$\mathrm{~b}$ & 2 & 2 & 0 & 3 \\
$\mathrm{c}$ & 1 & 3 & 1 & 2 \\
$\mathrm{~d}$ & 2 & 2 & 1 & 2 \\
\hline
\end{tabular}

\begin{tabular}{ccccc}
\hline \hline Alternative & $V_{1}^{*}$ & $V_{2}^{*}$ & $V_{3}^{*}$ & $\mathrm{MR}$ \\
\hline $\mathrm{a}$ & $\mathbf{2}$ & 2 & $\mathbf{7}$ & 2
\end{tabular}

\begin{tabular}{ccccc}
\hline \hline Alternative & $v_{1}^{*}$ & $v_{2}^{*}$ & $v_{3}^{*}$ & $v_{4}$ \\
\hline & 6 & 0 & 7 & 0
\end{tabular}

Profile $1 \cup$ Profile 2:

\begin{tabular}{lllll}
$\mathrm{a}$ & 6 & 0 & 7 & 0 \\
$\mathrm{~b}$ & 3 & 7 & 0 & 3 \\
$\mathrm{c}$ & 2 & 4 & 5 & 2 \\
$\mathrm{~d}$ & 2 & 2 & 1 & 8 \\
\hline
\end{tabular}

\begin{tabular}{ccccc}
\hline \hline Alternative & $V_{i}^{*}$ & $V_{2}^{*}$ & $V_{3}^{*}$ & $\mathrm{MR}$ \\
\hline $\mathrm{a}$ & $\mathbf{6}$ & 6 & $\mathbf{1 3}$ & 4 \\
$\mathrm{~b}$ & 3 & $\mathbf{1 0}$ & 10 & 3 \\
$\mathrm{c}$ & 2 & 6 & 11 & 4 \\
$\mathrm{~d}$ & 2 & 4 & 5 & 8 \\
\hline
\end{tabular}

Table 2 An example showing that aggregation with minimax regret does not satisfy consistency: assuming decreasing weights, alternative $a$ is picked as the minimax regret alternative in both profile 1 and profile 2 , but $b$ is selected by the rule when the two profiles are merged.

1. the set of undominated alternatives is the same in $p$ and $p^{\prime}$, and

2. the rank distributions of all alternatives that are undominated is the same in $p$ and $p^{\prime}$.

By inspecting Equation (22) and Equation (24) it is possible to see that the max regret of an alternative $x$ does not depend on the rank of dominated alternatives (indeed it depends only on the ranks of $x$ and on the ranks of some of the possible co-winners). Therefore MMR-Dec and MMR-Con satisfy IRDA.

Consistency (Young 1975), sometimes also called reinforcement, states that, if an alternative is the winner when considering two different population of voters, then the same alternative should be a winner when the two populations are merged together. Minimax regret does not satisfy consistency, as seen by the following counterexample.

Example 5 In Table 2 we present an example showing that aggregation with minimax regret does not satisfy the property of consistency: indeed in both profiles 1 and 2, the alternative a is the winner according to minimax regret. In the combined profile, however, $b$, and not $a$, is the winner.

We reckon that it is not that surprising that aggregation with minimax regret is not consistent: according to Young's axiomatization a rule that is neutral, anonymous and consistent should be a scoring rule (and clearly MMR-Dec and MMR-Con are not equivalent to any scoring rule).

The next property that we check is homogeneity, requiring that replicating the same profile several times lead to the same social choice. In terms of rank distribution, when a profile is replicated several times, the $v$ values are multiplied by $k: v_{j}^{x}\left[p^{\prime}\right]=k v_{j}^{x}[p]$, for all positions $j$ and for all alternatives $x$. It is a very easy exercise to verify that the pairwise max regret values are multiplied by $k$; for any $x$ and $y$,

$$
\operatorname{PMR}_{p^{\prime}}(x, y ; W)=k \operatorname{PMR}_{p}(x, y ; W)
$$

and that therefore

$$
\operatorname{MR}_{p^{\prime}}(x ; W)=k \operatorname{MR}_{p}(x ; W)
$$

leaving the winner, that is the alternative(s) associated with minimax regret, unchanged. Therefore MMR-Dec and MMR-Con are homogeneous.

Another property commonly studied in social choice is the independence from adding symmetric profiles (Merlin 2003): adding a symmetric profile (a profile where all permutations are present in the same number) should not change the result of the aggregation. Consider the profile $p^{\prime}$ obtained from the profile $p$ by adding a symmetric profile; we have

$$
v_{j}^{x}\left[p^{\prime}\right]=v_{j}^{x}[p]+\lambda \forall x \in A, \forall j
$$


and therefore $\operatorname{MR}_{p^{\prime}}(x ; W)=\operatorname{MR}_{p}(x ; W)$ for all $x \in A$, from which it follows $\operatorname{MMR}_{p^{\prime}}(W)=$ $\operatorname{MMR}_{p}(W)$; then our social choice functions give the same output in $p$ and $p^{\prime}$. We conclude that the property is satisfied by MMR-Dec and MMR-Con.

MMR-Dec and MMR-Con do not necessarily pick a Condorcet winner, in the case it exists. One may ask if the rules satisfy a weaker notion of Condorcet winner; the absolute majority property states that an alternative ranked first by a majority of the voters should be picked as the winner. In general, aggregation with minimax regret does not satisfy the property of absolute majority, unless in the trivial case with only two alternatives $(m=2)$.

We can, however, determine a condition on the number of times an alternative needs to be ranked in first position in order to ensure being chosen as a winner by MMR-Dec and MMR-Con. In particular, under decreasing weights, if an alternative is ranked first by at least two thirds of the voters, then this alternative is the single winner for MMR-Dec. For convex weights a smaller fraction of first position is required; for large number of alternatives this fraction is about two thirds as well.

Proposition 8 Let $p$ be the profile.

- When an alternative $x \in A$ is ranked first at least $\frac{2}{3} n$ times, then $\operatorname{MMR-Dec}(p)=\{x\}$.

In other words, if $x$ is such that $v_{1}^{x}>\frac{2}{3} n$ then $S^{*}\left(W^{D}\right)=\{x\}$

- When an alternative $x \in A$ is ranked first at least $\frac{2 m-2}{3 m-2} n$ times, then MMR-Con $(p)=\{x\}$.

In other words, if $x$ is such that $v_{1}^{x}>\frac{2 m-2}{3 m-2} n$ then $S^{*}\left(W^{C}\right)=\{x\}$.

We wrap up our discussion on the social choice properties of MMR-Dec and MMR-Con in the following theorem.

Theorem 3 The social choice functions MMR-Dec and MMR-Con satisfy anonymity, neutrality, unanimity, monotonicity, IRDA, homogeneity and independence from symmetric profiles.

\section{Numerical Tests with Real Data}

We perform a number of tests with real data; our intention is to provide some examples of regretbased aggregation (using the social choice functions MMR-Dec and MMR-Con) and to compare the results of the proposed aggregation method with some common voting rules (such as plurality, Borda, k-approval). We are also interested in numerically evaluating the size of the set of undominated alternatives and check how often there are undominated alternatives that are not possible winners.

We consider the rankings of the F1 race championship from 1961 to 2008, obtained from the PREFLIB data repository. ${ }^{6}$ In this context, an alternative is a driver and each ranking is the result of a race.

Undominated alternatives and possible winners The first part of the analysis consists in computing the number of undominated alternatives and that of possible winners.

We analyze the number of undominated alternatives and possible winners in Figure 5 . The number of drivers (corresponding to $m$ in our model) ranges from to 22 to 62 ; $n$, the number of races (not shown in the figure) ranges from to 8 to 19. The bar graph at the top partitions the drivers into Pareto optimal and Pareto dominated (a driver is Pareto dominated if there is another one that is better ranked in all races). The number of Pareto alternatives in each year is represented by the blue bar; for instance 16 drivers out of 54 are Pareto in the championship of 1961, while there are 17 Pareto optimal drivers out 22 in 2008 (the height of the blue and yellow bar corresponds to the total number of drivers in a given year).

We then analyze (using the propositions of Section 2.3) which drivers are undominated (with respect to weak dominance), and which drivers are possible winners, applying the concepts described in Section 2.4 (remember that a possible winner is necesseraly undominated). The second bar graph of Figure 5 shows the number of undominated alternatives and the number of possible winners in the hypothesis of decreasing weights, $W^{D}$.

\footnotetext{
${ }^{6}$ http://www.preflib.org/.
} 

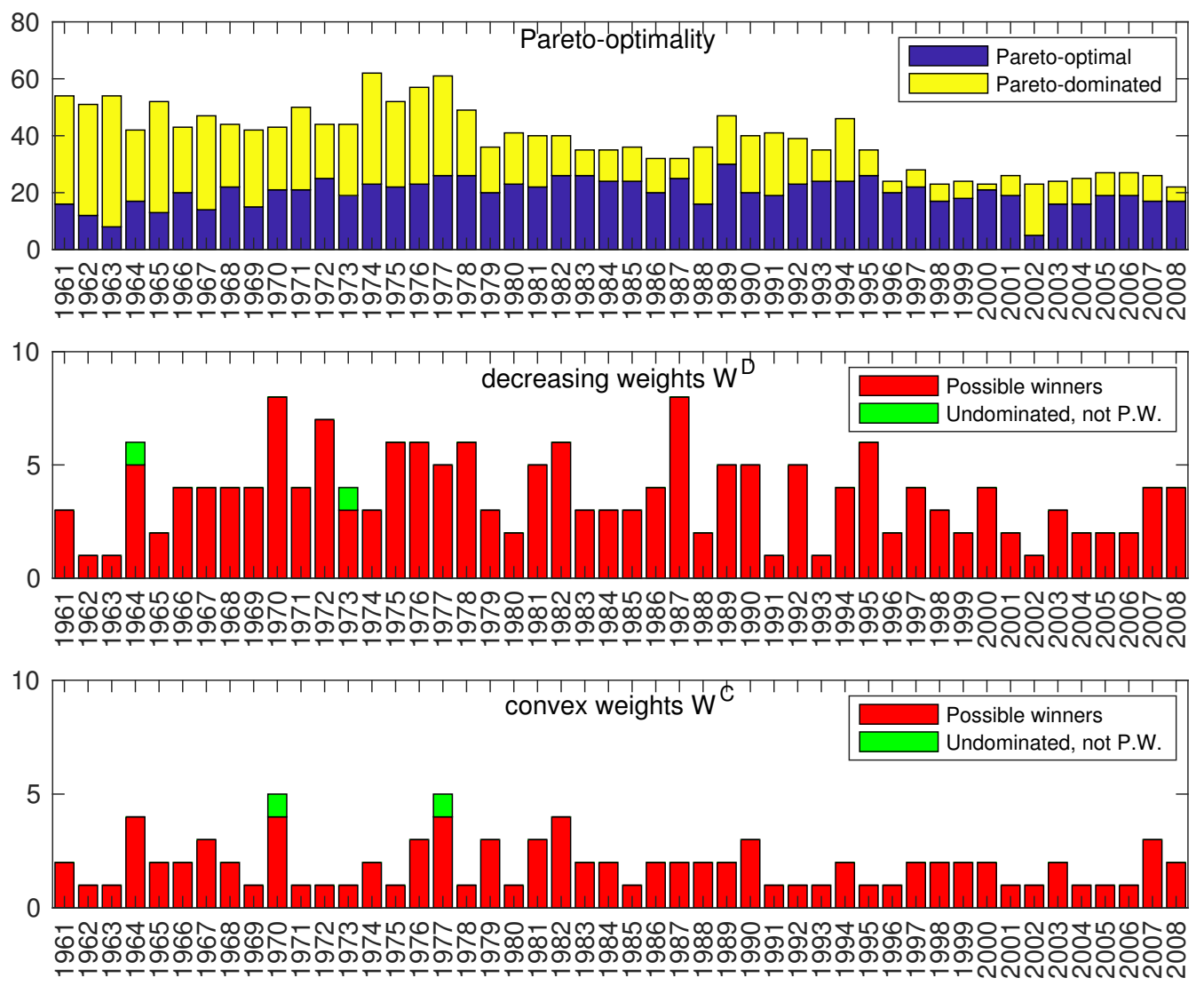

Fig. 5 Number of Pareto dominated and Pareto optimal alternatives (top chart), number of undominated alternatives and possible winners considering decreasing (middle chart) and convex (bottom char) scoring vectors, in actual rank data (F1 races).

We can observe that there are often several possible winners (alternatives that can win with an instantiation of the weights) but never more than 8 . In just 5 years there is a necessary winner $(1962,1963,1991,1993,2002)$; this happens when there is only one possible winner.

The third bar graph, at the bottom of Figure 5, shows that, when assuming convex scoring vectors, the number of possible winners is considerably reduced (and there is a necessary winner in several circumstances) but in most of the cases there is more than one possible winner.

We observed that there are sometimes undominated alternatives that are not possible winners, however this event is quite rare (it happens only two times when assuming $W^{D}$ and two times assuming $W^{C}$ ).

Winner determination with minimax regret We report some examples of using the aggregation method using minimax regret to determine a winner with an uncertain scoring vector. We consider again the F1 championship data and we determine which driver (or drivers in case of ties) is selected as winner according to the different aggregation methods. Table 3 shows, for the period 1987-2008, the scores of different drivers according to different methods (plurality, 3-approval, Borda, the point system used by F1 and minimax regret). In particular, the last two columns show max regret values when assuming either monotone or convex weights. Only drivers that are undominated in $W^{D}$ are shown; moreover a checkmark $(\checkmark)$ in the last column means that the driver is also undominated in $W^{C}$ (obviously since $W^{C} \subset W^{D}$, the undominated alternatives in $W^{C}$ are a subset of the undominated in $W^{D}$ ). For example, in the F1 championship of 2008, there are four possible winners (Hamilton, Kubica, Heidfeld and Massa) when assuming decreasing weights but only two (Hamilton and Massa) when assuming convex weights. When considering convex sequences, there is a tie between Hamilton and Massa with respect to max regret.

Remember that an alternative with max regret value of 0 is a necessary co-winner (see Proposition 6); this situation happens only three times (in 1991, 1993 and in 2002) when considering 


\begin{tabular}{|c|c|c|c|c|c|c|c|}
\hline & Alternative & Plurality & 3-approval & Borda & $\mathrm{F} 1_{2010}$ & $\operatorname{MR}\left(\cdot, W^{D}\right)$ & $\operatorname{MR}\left(\cdot, W^{C}\right)$ \\
\hline \multirow[t]{4}{*}{1986} & Alain Prost & 4.00 & 11.00 & 13.87 & 9.32 & 1.00 & $1.00 \checkmark$ \\
\hline & Nlson Piquet & 4.00 & 10.00 & 13.61 & 8.68 & 2.00 & 1.00 \\
\hline & Ren Arnoux & 0.00 & 0.00 & 11.68 & 3.08 & 11.00 & 7.75 \\
\hline & Nigel Mansell & 5.00 & 9.00 & 13.26 & 8.92 & 2.00 & $0.83 \checkmark$ \\
\hline \multirow{8}{*}{1987} & Gerhard Berger & 2.00 & 3.00 & 10.19 & 4.64 & 8.00 & 5.33 \\
\hline & Michele Alboreto & 0.00 & 3.00 & 9.58 & 2.56 & 9.00 & 7.43 \\
\hline & Thierry Boutsen & 0.00 & 1.00 & 10.81 & 3.00 & 10.00 & 8.00 \\
\hline & Jonathan Palmer & 0.00 & 0.00 & 11.10 & 2.40 & 11.00 & 8.83 \\
\hline & Ayrton Senna & 2.00 & 8.00 & 13.42 & 7.68 & 4.00 & 4.00 \\
\hline & Nlson Piquet & 3.00 & 11.00 & 13.48 & 9.12 & 3.00 & $3.00 \checkmark$ \\
\hline & Teo Fabi & 0.00 & 1.00 & 9.87 & 2.60 & 10.00 & 8.25 \\
\hline & Nigel Mansell & 6.00 & 7.00 & 11.16 & 7.32 & 5.00 & $3.00 \checkmark$ \\
\hline \multirow[t]{2}{*}{1988} & Alain Prost & 7.00 & 14.00 & 14.83 & 12.04 & 1.00 & $1.00 \checkmark$ \\
\hline & Ayrton Senna & 8.00 & 11.00 & 14.69 & 11.00 & 3.00 & $1.80 \checkmark$ \\
\hline \multirow[t]{5}{*}{1989} & Thierry Boutsen & 2.00 & 5.00 & 12.80 & 5.16 & 8.00 & 5.40 \\
\hline & Alessandro Nannini & 1.00 & 4.00 & 12.43 & 4.76 & 8.00 & 6.00 \\
\hline & Riccardo Patrese & 0.00 & 6.00 & 13.11 & 5.44 & 6.00 & 6.00 \\
\hline & Ayrton Senna & 6.00 & 7.00 & 13.13 & 6.96 & 6.00 & $4.20 \mathrm{r}$ \\
\hline & Alain Prost & 4.00 & 11.00 & 14.50 & 9.80 & 2.00 & $2.00 \checkmark$ \\
\hline \multirow{5}{*}{1990} & Nlson Piquet & 2.00 & 4.00 & 13.82 & 6.76 & 7.00 & $5.33 \checkmark$ \\
\hline & Nigel Mansell & 1.00 & 5.00 & 12.23 & 4.80 & 6.00 & 5.00 \\
\hline & Alain Prost & 5.00 & 9.00 & 13.54 & 9.00 & 3.00 & $1.33 \checkmark$ \\
\hline & Gerhard Berger & 0.00 & 7.00 & 13.36 & 6.28 & 6.00 & 6.00 \\
\hline & Ayrton Senna & 6.00 & 11.00 & 13.51 & 9.24 & 3.00 & $0.55 \checkmark$ \\
\hline 1991 & Ayrton Senna & 7.00 & 12.00 & 15.05 & 11.48 & 0.00 & $0.00 \checkmark$ \\
\hline \multirow[t]{5}{*}{1992} & Michael Schumacher & 1.00 & 8.00 & 13.37 & 7.24 & 8.00 & 8.00 \\
\hline & J J Lehto & 0.00 & 0.00 & 11.29 & 0.92 & 12.00 & 11.50 \\
\hline & Riccardo Patrese & 1.00 & 9.00 & 13.24 & 7.08 & 8.00 & 8.00 \\
\hline & Nigel Mansell & 9.00 & 12.00 & 14.00 & 11.16 & 1.00 & $0.00 \checkmark$ \\
\hline & Michele Alboreto & 0.00 & 0.00 & 12.29 & 3.00 & 12.00 & 11.25 \\
\hline 1993 & Alain Prost & 7.00 & 12.00 & 14.62 & 10.84 & 0.00 & $0.00 \checkmark$ \\
\hline \multirow[t]{4}{*}{1994} & Olivier Panis & 0.00 & 1.00 & 12.80 & 2.20 & 10.00 & 9.00 \\
\hline & Christian Fittipaldi & 0.00 & 0.00 & 12.24 & 1.68 & 11.00 & 9.43 \\
\hline & Damon Hill & 6.00 & 11.00 & 14.38 & 10.08 & 2.00 & $2.00 \checkmark$ \\
\hline & Michael Schumacher & 8.00 & 10.00 & 12.64 & 9.44 & 3.00 & $1.74 \checkmark$ \\
\hline 1995 & Damon Hill & 4.00 & 9.00 & 13.62 & 7.84 & 5.00 & 5.00 \\
\hline & Mika Salo & 0.00 & 0.00 & 11.88 & 2.00 & 11.00 & 10.25 \\
\hline & Johnny Herbert & 2.00 & 4.00 & 13.82 & 6.72 & 7.00 & 7.00 \\
\hline & Gerhard Berger & 0.00 & 6.00 & 12.91 & 4.88 & 10.00 & 9.50 \\
\hline & Michael Schumacher & 9.00 & 11.00 & 14.47 & 10.72 & 2.00 & $0.00 \checkmark$ \\
\hline & Rubens Barrichello & 0.00 & 1.00 & 11.41 & 2.32 & 10.00 & 9.40 \\
\hline 1996 & Jacques Villeneuve & 4.00 & 11.00 & 13.17 & 9.20 & 4.00 & 4.00 \\
\hline & Damon Hill & 8.00 & 10.00 & 13.35 & 10.32 & 1.00 & $0.00 \checkmark$ \\
\hline 1997 & Jacques Villeneuve & 7.00 & 8.00 & 13.30 & 8.88 & 2.00 & $1.17 \checkmark$ \\
\hline & Mika Salo & 0.00 & 0.00 & 9.67 & 0.68 & 12.00 & 9.56 \\
\hline & Jean Alesi & 0.00 & 5.00 & 12.96 & 5.72 & 7.00 & 7.00 \\
\hline & Michael Schumacher & 5.00 & 8.00 & 13.48 & 9.16 & 3.00 & $2.00 \checkmark$ \\
\hline 1998 & Michael Schumacher & 6.00 & 11.00 & 13.27 & 9.68 & 2.00 & $2.00 \checkmark$ \\
\hline & Mika Hkkinen & 8.00 & 11.00 & 13.18 & 10.84 & 2.00 & $0.11 \checkmark$ \\
\hline & Eddie Irvine & 0.00 & 8.00 & 12.23 & 6.96 & 8.00 & 8.00 \\
\hline 1999 & Mika Hkkinen & 5.00 & 10.00 & 12.17 & 8.64 & 4.00 & $2.40 \checkmark$ \\
\hline & Eddie Irvine & 4.00 & 9.00 & 13.91 & 9.48 & 1.00 & $1.00 \checkmark$ \\
\hline 2000 & Mika Hkkinen & 4.00 & 11.00 & 14.05 & 10.32 & 5.00 & $5.00 \checkmark$ \\
\hline & Michael Schumacher & 9.00 & 12.00 & 13.68 & 11.44 & 2.00 & $0.40 \checkmark$ \\
\hline & David Coulthard & 3.00 & 11.00 & 13.77 & 9.28 & 6.00 & 6.00 \\
\hline & Ralf Schumacher & 0.00 & 3.00 & 10.36 & 4.20 & 11.00 & 10.00 \\
\hline 2001 & Michael Schumacher & 9.00 & 14.00 & 15.36 & 13.08 & 1.00 & $0.00 \checkmark$ \\
\hline & Jean Alesi & 0.00 & 0.00 & 11.24 & 2.36 & 15.00 & 13.20 \\
\hline 2002 & Michael Schumacher & 11.00 & 17.00 & 16.68 & 15.20 & 0.00 & $0.00 \checkmark$ \\
\hline 2003 & Kimi Rikkonen & 1.00 & 10.00 & 12.61 & 8.52 & 5.00 & $5.00 \mathrm{r}$ \\
\hline & Juan Pablo Montoya & 2.00 & 9.00 & 12.39 & 7.84 & 4.00 & 4.00 \\
\hline & Michael Schumacher & 6.00 & 8.00 & 13.74 & 9.44 & 2.00 & $0.50 \checkmark$ \\
\hline 2004 & Michael Schumacher & 13.00 & 15.00 & 16.79 & 14.68 & 1.00 & $0.00 \checkmark$ \\
\hline & Rubens Barrichello & 2.00 & 14.00 & 15.79 & 10.84 & 11.00 & 11.00 \\
\hline 2005 & Rubens Barrichello & 0.00 & 4.00 & 13.58 & 4.12 & 12.00 & 10.20 \\
\hline & Fernando Alonso & 7.00 & 15.00 & 17.04 & 12.88 & 1.00 & $0.00 \checkmark$ \\
\hline 2006 & Nick Heidfeld & 0.00 & 1.00 & 11.69 & 2.72 & 14.00 & 12.33 \\
\hline & Fernando Alonso & 7.00 & 14.00 & 16.19 & 12.84 & 1.00 & $0.00 \checkmark$ \\
\hline 2007 & Lewis Hamilton & 4.00 & 12.00 & 15.00 & 10.60 & 2.00 & $2.00 \checkmark$ \\
\hline & Kimi Rikkonen & 6.00 & 12.00 & 14.84 & 10.88 & 2.00 & $0.46 \checkmark$ \\
\hline & Fernando Alonso & 4.00 & 12.00 & 14.96 & 10.64 & 2.00 & $2.00 \checkmark$ \\
\hline & Heikki Kovalainen & 0.00 & 1.00 & 11.96 & 3.48 & 11.00 & 9.33 \\
\hline 2008 & Lewis Hamilton & 5.00 & 10.00 & 14.43 & 9.72 & 2.00 & $1.00 \checkmark$ \\
\hline & Robert Kubica & 1.00 & 7.00 & 14.00 & 7.40 & 5.00 & 5.00 \\
\hline & Nick Heidfeld & 0.00 & 4.00 & 13.29 & 6.00 & 6.00 & 6.00 \\
\hline & Felipe Massa & 6.00 & 10.00 & 13.62 & 9.60 & 4.00 & $1.00 \checkmark$ \\
\hline
\end{tabular}

Table 3 The scores obtained by undominated alternatives in F1 championships (years 1986-2008) with different aggregation methods. 


\begin{tabular}{lccccccc}
\hline Disagreement & $\mathrm{F} 1$ & plurality & 3-approval & 5-approval & Borda & $\mathrm{MR}\left(\cdot ; W^{D}\right)$ & $\mathrm{MR}\left(\cdot ; W^{C}\right)$ \\
\hline F1 & 0 & 0.41 & 0.23 & 0.30 & 0.29 & 0.17 & 0.17 \\
plurality & 0.41 & 0 & 0.47 & 0.60 & 0.55 & 0.43 & 0.28 \\
3-approval & 0.23 & 0.47 & 0 & 0.35 & 0.40 & 0.26 & 0.34 \\
5-approval & 0.30 & 0.60 & 0.35 & 0 & 0.35 & 0.31 & 0.38 \\
Borda & 0.29 & 0.55 & 0.40 & 0.35 & 0 & 0.28 & 0.39 \\
$\operatorname{MR}\left(\cdot ; W^{D}\right)$ & 0.17 & 0.43 & 0.26 & 0.31 & 0.28 & 0 & 0.18 \\
$\operatorname{MR}\left(\cdot ; W^{C}\right)$ & 0.17 & 0.28 & 0.34 & 0.38 & 0.39 & 0.18 & 0 \\
\hline
\end{tabular}

Table 4 Average disagreement (with respect to winner) between the different rank aggregation methods (F1 championship dataset; years 1961-2008).

decreasing weights, but it happens several times when considering the uncertainty being over convex weights.

We remarked before (see Proposition 5 and as well discussion in Section 4) that it is theoretically possible that relations of weak dominance hold between alternatives tied at the minimax regret value (i.e. between alternatives that minimize max regret). However, we never observed such phenomenon in this dataset: the winners according to minimax regret are always strongly undominated.

Comparison of different aggregation methods We now want to analyze how often different social choice functions pick a different winner. We compare our methods based on max regret, that is the social choice functions MMR-Dec and MMR-Con (considering no discriminative thresholds), with plurality, 3-approval, 5-approval, Borda and ranking with the true $\mathrm{F} 1$ point system ${ }^{7}$. In Table 4 we analyze how often the different methods disagree on picking the winner (considering all years 1961-2008). We compute the disagreement between two sets of winners as the cardinality of the symmetric set difference normalized by the cardinality of the union; in this way we are able to handle the case where an aggregation method may give the same score to more than an alternative, returning multiple winners. While often all methods pick the same winners, there is a considerable difference between some of the methods.

The two methods based on minimax regret (one assuming decreasing weights in $W^{D}$ and the other assuming convex weights in $W^{C}$ ) pick a different winner $18 \%$ of the times. Minimax regret is more "similar" to Borda when considering $W^{D}$ rather than $W^{C}$; while minimax regret assuming the weights in $W^{C}$ is more similar to plurality than when using $W^{D}$ (this fact is not surprising since plurality is itself a convex rule). Both methods based on regret agree about $83 \%$ of the times with the aggregation using the real F1 point system.

\section{Conclusions}

While scoring rules are often used for aggregating rankings, determining the scoring vector (the weights associated to each position) is a crucial step, since different scoring vectors may induce picking different winners.

In this paper we considered how to aggregate the input rankings by assuming a scoring vector with uncertain weights, proposing to adopt the minimax regret criterion. Minimax regret ensures that the decision is robust with respect to the full range of possible choices for the scoring vector.

We assume that we are given a set of possible scoring vectors addressing the case of decreasing weights and convex sequences of decreasing weights. We also considered the possibility that the decision maker may impose some "discriminative thresholds" imposing a minimum separation between the weights. The main contributions of this paper are the characterization of max regret in terms of cumulative ranks (giving efficient way to compute the regret optimal alternative that does not require linear programming) and the analysis of the properties of minimax regret from the point of view of social choice theory.

\footnotetext{
7 Several different versions have been adopted over the years; here we choose to compare only with respect to the point system used in 2010 .
} 
We mention some additional related works. Recently researchers in computational social choice theory have been considering methods for dealing with partially specified preference profiles; for example, possible and necessary winners when aggregating partial orders (Xia and Conitzer 2011). While we considered weights associated to positions, Baumeister et al. (2012) have considered aggregation rules with weights attached to individual voters and provided methods for computing possible winners. Some authors have considered machine learning methods in relation to scoring rules: Procaccia et al. (2009) studied how a scoring vector can be learned from examples, while Haghtalab et al. (2018) considered, in a setting of repeated social choice, how to learn the weights attached to voters.

Finally, we mention that it has been questioned (Bossert and Suzumura 2018) the idea that the positional scores have to be aggregated using a sum; in particular, the OWA operator has been considered (Goldsmith et al. 2014; García-Lapresta and Martínez-Panero 2017) as an alternative aggregator.

Acknowledgements This work was partially supported by the ANR project Cocorico-CoDec. The author thanks two anonymous reviewers for helpful comments. Moreover, the author would like to thank Jerome Lang for several comments on an early version of this paper, Stefano Moretti for pointing out some typos, and Patrice Perny for discussion on dominance relations and possible winners.

\section{References}

Baumeister D, Roos M, Rothe J, Schend L, Xia L (2012) The possible winner problem with uncertain weights. In: ECAI 2012 - 20th European Conference on Artificial Intelligence. Montpellier, France, August 27-31 , 2012, pp 133-138, DOI 10.3233/978-1-61499-098-7-133

Bossert W, Suzumura K (2018) Positionalist voting rules: A general definition and axiomatic characterizations. Tech. rep., URL http://pages.videotron.com/wbossert/positionalist_dec18.pdf

Boutilier C, Patrascu R, Poupart P, Schuurmans D (2006) Constraint-based optimization and utility elicitation using the minimax decision criterion. Artifical Intelligence 170(8-9):686-713

Braziunas D, Boutilier C (2008) Elicitation of factored utilities. AI Magazine 29(4):79-92

Cook WD, Kress M (1990) A data envelopment model for aggregating preference rankings. Management Science 36(11):1302-1310

Ehrgott M (2005) Multicriteria Optimization (2. ed.). Springer, DOI 10.1007/3-540-27659-9, URL https://doi. org/10.1007/3-540-27659-9

Fishburn P, Gehrlein W (1976) Borda's rule, positional voting, and Condorcet's simple majority principle. Public Choice 28:79-88

Fishburn PC, Vickson RG (1978) Theoretical foundations of stochastic dominance. In: Whitmore GA, Findlay MC (eds) Stochastic Dominance, D.C. Heath and Co., Lexington, MA, USA, pp 37-113

Foroughi A, Tamiz M (2005) An effective total ranking model for a ranked voting system. Omega 33(6):491 - 496, DOI https://doi.org/10.1016/j.omega.2004.07.013, URL http://www.sciencedirect.com/science/article/ $\mathrm{pii/S0305048304001070}$

French S (ed) (1986) Decision Theory: An Introduction to the Mathematics of Rationality. Halsted Press, New York, NY, USA

García-Lapresta JL, Martínez-Panero M (2017) Positional voting rules generated by aggregation functions and the role of duplication. Int J Intell Syst 32(9):926-946, DOI 10.1002/int.21877

Goldsmith J, Lang J, Mattei N, Perny P (2014) Voting with rank dependent scoring rules. In: Proceedings of the Twenty-Eighth AAAI Conference on Artificial Intelligence, July 27 -31, 2014, Québec City, Québec, Canada., pp 698-704

Green RH, Doyle JR, Cook WD (1996) Preference voting and project ranking using dea and cross-evaluation. European Journal of Operational Research 90(3):461 - 472, DOI https://doi.org/10.1016/0377-2217(95)00039-9

Haghtalab N, Noothigattu R, Procaccia AD (2018) Weighted voting via no-regret learning. In: Proceedings of the Thirty-Second AAAI Conference on Artificial Intelligence, (AAAI-18), New Orleans, Louisiana, USA, February 2-7, 2018, pp 1055-1062

Hashimoto A (1997) A ranked voting system using a dea/ar exclusion model: A note. European Journal of Operational Research 97(3):600 - 604, DOI https://doi.org/10.1016/S0377-2217(96)00281-0

Hazen GB (1986) Partial information, dominance, and potential optimality in multiattribute utility theory. Operations Research 34(2):296-310, DOI 10.1287/opre.34.2.296

Khodabakhshi M, Aryavash K (2015) Aggregating preference rankings using an optimisticpessimistic approach. Computers \& Industrial Engineering 85:13 - 16, DOI https://doi.org/10.1016/j.cie.2015.02.030

Konczak K, Lang J (2005) Voting procedures with incomplete preferences. In: in Proc. IJCAI-05 Multidisciplinary Workshop on Advances in Preference Handling

Kouvelis P, Yu G (1997) Robust Discrete Optimization and Its Applications. Kluwer, Dordrecht

Llamazares B (2016) Ranking candidates through convex sequences of variable weights. Group Decision and Negotiation 25:567-584, DOI 10.1007/s10726-015-9452-8 
Llamazares B, Peña T (2009) Preference aggregation and DEA: an analysis of the methods proposed to discriminate efficient candidates. European Journal of Operational Research 197(2):714-721, DOI 10.1016/j.ejor.2008.06.031, URL https://doi.org/10.1016/j.ejor.2008.06.031

Llamazares B, Peña T (2013) Aggregating preferences rankings with variable weights. European Journal of Operational Research 230(2):348-355, DOI 10.1016/j.ejor.2013.04.013, URL https://doi.org/10.1016/j.ejor. 2013. 04.013

Llamazares B, Peña T (2015a) Positional voting systems generated by cumulative standings functions. Group Decision and Negotiation 24(5):777-801, DOI 10.1007/s10726-014-9412-8

Llamazares B, Peña T (2015b) Scoring rules and social choice properties: some characterizations. Theory and Decision 78(3):429-450, DOI 10.1007/s11238-014-9429-0

$\mathrm{Lu} \mathrm{T}$, Boutilier C (2011) Robust approximation and incremental elicitation in voting protocols. In: Proceedings of IJCAI 2011, pp 287-293

Merlin V (2003) The axiomatic characterizations of majority voting and scoring rules. Mathématiques et sciences humaines Mathematics and social sciences (163)

Pearman AD (1993) Establishing dominance in multiattribute decision making using an ordered metric method. Journal of the Operational Research Society 44(5):461-469

Peterson M (2017) An Introduction to Decision Theory, 2nd edn. Cambridge Introductions to Philosophy, Cambridge University Press, DOI 10.1017/9781316585061

Procaccia AD, Zohar A, Peleg Y, Rosenschein JS (2009) The learnability of voting rules. Artif Intell 173(12-13):11331149, DOI 10.1016/j.artint.2009.03.003, URL https://doi.org/10.1016/j.artint.2009.03.003

Salo A (1995) Interactive decision aiding for group decision support. European Journal of Operational Research 84:134-149, DOI 10.1016/0377-2217(94)00322-4

Salo A, Hämäläinen RP (2001) Preference ratios in multiattribute evaluation (PRIME)-elicitation and decision procedures under incomplete information. IEEE Trans on Systems, Man and Cybernetics 31(6):533-545

Savage LJ (1954) The Foundations of Statistics. Wiley, New York

Stein WE, Mizzi PJ, Pfaffenberger RC (1994) A stochastic dominance analysis of ranked voting systems with scoring. European Journal of Operational Research 74(1):78 - 85, DOI https://doi.org/10.1016/0377-2217(94)90205-4, URL http://www.sciencedirect.com/science/article/pii/0377221794902054

Viappiani P (2018) Positional scoring rules with uncertain weights. In: Proc. od Scalable Uncertainty Management - 12th International Conference, SUM 2018, Milan, Italy, October 3-5, 2018, pp 306-320

Weber M (1987) Decision making with incomplete information. European Journal of Operational Research 28(1):44 - 57, DOI https://doi.org/10.1016/0377-2217(87)90168-8

Xia L, Conitzer V (2011) Determining possible and necessary winners given partial orders. J Artif Intell Res 41:25-67

Young HP (1974) An axiomatization of Borda's rule. Journal of Economic Theory 9:43-52

Young HP (1975) Social choice scoring functions. SIAM Journal on Applied Mathematics 28(4):824-838, URL http://www.jstor.org/stable/2100365

Zwicker WS (2016) Introduction to the theory of voting. In: Brandt F, Conitzer V, Endriss U, Lang J, Procaccia AD (eds) Handbook of Computational Social Choice, Cambridge University Press, pp 23-56, DOI 10.1017/ CBO9781107446984.003, URL https://doi.org/10.1017/CB09781107446984.003

\section{A Appendix: Proofs}

We omit the proof of Proposition 3 that is trivial.

Proposition 4 Let $x, y \in A$. The following statements connect max regret and dominance relations:

1. For an arbitrary set $W$ of scoring vectors:

If $x$ weakly dominates $y$, then $\operatorname{MR}(x ; W) \leq \mathrm{MR}(y ; W)$;

if $x$ strongly dominates $y$, then $\operatorname{MR}(x ; W)<\operatorname{MR}(y ; W)$.

2. Moreover, when considering decreasing weights, i.e. the scoring vector belongs to $W^{D}$ :

If $V^{x} \succeq V^{y}$ then $\operatorname{MR}\left(x ; W^{D}\right) \leq \operatorname{MR}\left(y ; W^{D}\right)$;

if $V^{x} \succ V^{y}$ then $\operatorname{MR}\left(x ; W^{D}\right)<\operatorname{MR}\left(y ; W^{D}\right)$.

3. Finally, when considering convex weights, i.e. the scoring vector belongs to $W^{C}$ :

If $\mathcal{V}^{x} \succeq \mathcal{V}^{y}$ then $\operatorname{MR}\left(x ; W^{C}\right) \leq \operatorname{MR}\left(y ; W^{C}\right)$;

if $\mathcal{V}^{x} \succ \mathcal{V}^{y}$ then $\operatorname{MR}\left(x ; W^{C}\right)<\operatorname{MR}\left(y ; W^{C}\right)$.

Proof 1) If $x$ weakly dominates $y$, by definition $\forall w \in W \quad s(x ; w) \geq s(y ; w)$ (Definition 3). For any $z \in A$, we have that:

$$
\operatorname{PMR}(x, z ; W)=\max _{w \in W}\{s(z ; w)-s(x ; w)\} \leq \max _{w \in W}\{s(z ; w)-s(y ; w)\}=\operatorname{PMR}(y, z ; W) .
$$

Therefore $\operatorname{MR}(x ; W)=\max _{z \in A} \operatorname{PMR}(x, z ; W) \leq \max _{z \in A} \operatorname{PMR}(y, z ; W)=\operatorname{MR}(y ; W)$.

2) The result follows directly from part 1 of this proposition and Proposition 1

3) The result follows directly from part 1 of this proposition and Proposition 2.

Proposition 5 The followings statements hold:

1. For any $x \in S^{*}(W), x$ is weakly undominated, i.e. there is no $y \in A$ such that $y$ strongly dominates $x$.

2. For all $x \in S^{*}(W)$, there is no $y \in A \backslash S^{*}(W)$ such that $y$ weakly dominates $x$. 
Proof 1) Let $x \in S^{*}(W)$, by definition $x \in \arg \min _{z \in A} \operatorname{MR}(z ; W)$. Now assume $y$ strongly dominates $x$. Then, by Proposition 4 , we have $\operatorname{MR}(y ; W)<\operatorname{MR}(x ; W)$, but this is absurd.

2) Let $x \in S^{*}(W)$. Now assume $y \in A \backslash S^{*}(W)$ and that $y$ weakly dominates $x$. Then, by Proposition 4 , we have $\operatorname{MR}(y ; W) \leq \operatorname{MR}(x ; W)$ and therefore $\operatorname{MR}(y ; W)=\operatorname{MMR}(W)$, meaning $y \in S^{*}(W)$. We obtain a contradiction.

Proposition 6 Let $x \in A$. The following statements hold:

1. Alternative $x$ is a necessary co-winner if and only if $\mathrm{MR}(x ; W)=0$.

2. If $x$ is a necessary winner then $\operatorname{MR}(x ; W)=0$ and $S^{*}(W)=\{x\}$.

3. Alternative $x$ is a necessary winner if and only if $\operatorname{PMR}(x, z ; W)<0$ for all $z \in A \backslash\{x\}$.

Proof 1. $x$ is a necessary co-winner $\Longleftrightarrow s(x ; w) \geq s(z ; w) \forall z \in A, \forall w \in W \Longleftrightarrow \operatorname{PMR}(x, z ; W) \leq 0$ for all $z \in A$ $\Longleftrightarrow \operatorname{MR}(x ; W)=0$.

2. If $x$ is a necessary winner then it is also a necessary co-winner, and part 1 of this proposition implies $\mathrm{MR}(x ; W)=$ 0 , and $x \in S^{*}(W)$. Let $y \in A \backslash\{x\}$. Since $x$ is a necessary winner, $s(x ; w)>s(y ; w)$ for all $w \in W$, therefore $\operatorname{PMR}(y, x ; W)>0$ and $\operatorname{MR}(y ; W) \geq \operatorname{PMR}(y, x ; W)>0$. Hence $S^{*}(W)=\{x\}$.

3. $x$ is a necessary winner $\Longleftrightarrow s(x ; w)>s(z ; w) \forall z \in A \backslash\{x\}, \forall w \in W \Longleftrightarrow \operatorname{PMR}(x, z ; W)<0$ for all $z \in A \backslash\{x\}$

Lemma 1 In the case of decreasing weights, for any pairs $x, y \in A$, it holds

$$
\operatorname{PMR}\left(x, y ; W^{D}\right)=\max _{j \in \llbracket m-1 \rrbracket}\left\{V_{j}^{y}-V_{j}^{x}\right\} .
$$

Proof By noticing that the optimal solution of a bounded linear program is attained in one of the vertices, we derive

$$
\begin{aligned}
\operatorname{PMR}\left(x, y ; W^{D}\right) & =\max \left\{\sum_{j=1}^{m}\left[w_{j} v_{j}^{y}-w_{j} v_{j}^{x}\right] \mid 1=w_{1} \geq w_{2} \geq \ldots \geq w_{m-1} \geq w_{m}=0\right\}= \\
& =\max \left\{\sum_{j=1}^{m-1} \delta_{j}\left(V_{j}^{y}-V_{j}^{x}\right) \mid \delta_{1} \geq 0, \ldots, \delta_{m-1} \geq 0 \wedge \sum_{j=1}^{m-1} \delta_{j}=1\right\}= \\
& =\max \left\{\sum_{j=1}^{m-1} \delta_{j}\left(V_{j}^{y}-V_{j}^{x}\right) \mid \delta \in\{\operatorname{oneat}(1), \ldots, \operatorname{oneat}(\mathrm{m}-1)\}\right\}= \\
& =\max _{j \in \llbracket m-1 \rrbracket}\left\{V_{j}^{y}-V_{j}^{x}\right\}
\end{aligned}
$$

where oneat $(\mathrm{j})$ is the vector with 0 everywhere except in position $j$ where the value is 1 .

Theorem 1 For any alternative $x \in A$ it holds

$$
\operatorname{MR}\left(x ; W^{D}\right)=\max _{j \in \llbracket m-1 \rrbracket}\left\{V_{j}^{*}-V_{j}^{x}\right\}
$$

where $V_{k}^{*}=\max _{x \in A} V_{k}^{x}$

Proof We use Lemma 1 and substitute Equation (21) into the formula of MR( $x$ ) given by Equation (17):

$\operatorname{MR}\left(x ; W^{D}\right)=\max _{y \in A} \max _{j \in \llbracket m-1 \rrbracket}\left\{V_{j}^{y}-V_{j}^{x}\right\}=\max _{j \in \llbracket m-1 \rrbracket} \max _{y \in A}\left\{V_{j}^{y}-V_{j}^{x}\right\}=\max _{j \in \llbracket m-1 \rrbracket}\left\{\max _{y \in A}\left\{V_{j}^{y}\right\}-V_{j}^{x}\right\}=\max _{j \in \llbracket m-1 \rrbracket}\left\{V_{j}^{*}-V_{j}^{x}\right\}$.

Lemma 2 Assuming convex weights, the pairwise max regret of an alternative $x$ against $y$ can be computed as follows:

$$
\operatorname{PMR}\left(x, y ; W^{C}\right)=\max _{j \in \llbracket m-1 \rrbracket}\left\{\frac{\mathcal{V}_{j}^{y}-\mathcal{V}_{j}^{x}}{j}\right\} .
$$

Proof The proof is similar to that of Lemma 1. The pairwise max regret can be computed using the following linear program.

$$
\begin{aligned}
\operatorname{PMR}\left(x, y ; W^{C}\right)=\max & \sum_{j=1}^{m-1} \phi_{j}\left(\mathcal{V}_{j}^{y}-\mathcal{V}_{j}^{x}\right) \\
\text { s.t. } & \sum_{j=1}^{m-1} j \phi_{j}=1 \\
& \phi_{1} \geq 0, \ldots, \phi_{m-1} \geq 0
\end{aligned}
$$

Let oneat $(\mathrm{j})$ be the vector with 0 everywhere except in position $j$ where the value is 1 .

We know from the theory of linear programming that the optimum is attained in one of the vertices. Therefore, the optimal $\phi$ must be of the type $j$. oneat $(j)$, that is $(1,0, \ldots, 0),(0,0.5,0, \ldots, 0), \ldots,\left(0, \ldots, 0, \frac{1}{m-1}\right)$. The corresponding optimal $\delta$ is among $(1,0, \ldots, 0),\left(\frac{1}{2}, \frac{1}{2}, 0, \ldots, 0\right),\left(\frac{1}{3}, \frac{1}{3}, \frac{1}{3}, 0, \ldots, 0\right)$, etc.

$$
\operatorname{PMR}\left(x, y ; W^{C}\right)=\max \left\{\sum_{j=1}^{m-1} \phi_{j}\left(\mathcal{V}_{j}^{y}-\mathcal{V}_{j}^{x}\right) \mid \phi \in\left\{\frac{\operatorname{oneat}(\mathrm{j})}{j}\right\}_{j=1}^{m-1}\right\}=\max _{j \in \llbracket m-1 \rrbracket} \frac{\mathcal{V}_{j}^{y}-\mathcal{V}_{j}^{x}}{j}
$$


Theorem 2 Assuming convex weights, the max regret of alternative $x$ can be computed as follows:

$$
\operatorname{MR}\left(x ; W^{C}\right)=\max _{j \in \llbracket m-1 \rrbracket}\left\{\frac{\mathcal{V}_{j}^{*}-\mathcal{V}_{j}^{x}}{j}\right\}
$$

where $\mathcal{V}_{j}^{*}=\max _{x \in A} \mathcal{V}_{j}^{x}$

Proof The result follows by using Lemma 2 in order to substitute Equation (23) in Equation (17); we then exchange the order of the two max.

$$
\operatorname{MR}\left(x ; W^{C}\right)=\max _{y \in A} \max _{j \in \llbracket m-1 \rrbracket}\left\{\frac{\mathcal{V}_{j}^{y}-\mathcal{V}_{j}^{x}}{j}\right\}=\max _{j \in \llbracket m-1 \rrbracket} \max _{y \in A}\left\{\frac{\mathcal{V}_{j}^{y}-\mathcal{V}_{j}^{x}}{j}\right\}=\max _{j \in \llbracket m-1 \rrbracket}\left\{\frac{\mathcal{V}_{j}^{*}-\mathcal{V}_{j}^{x}}{j}\right\} .
$$

Proposition 7 For any pair of alternatives $x, y \in A$, it holds

$$
\operatorname{PMR}\left(x, y ; W^{D, t}\right)=\left[\left(1-\sum_{j=1}^{m-1} t_{j}\right) \max _{j \in \llbracket m-1 \rrbracket}\left\{V_{j}^{y}-V_{j}^{x}\right\}\right]+\sum_{j=1}^{m-1} t_{j}\left(V_{j}^{y}-V_{j}^{x}\right)
$$

or, equivalently,

$$
\operatorname{PMR}\left(x, y ; W^{D, t}\right)=\left[\left(1-\sum_{j=1}^{m-1} t_{j}\right) \operatorname{PMR}\left(x, y ; W^{D}\right)\right]+\sum_{j=1}^{m-1} t_{j}\left(V_{j}^{y}-V_{j}^{x}\right)
$$

where $\operatorname{PMR}\left(x, y ; W^{D}\right)$ is pairwise max regret computed with no discriminative thresholds.

Proof We let $\hat{\delta}_{j}$ to be the slack between the value of $\delta_{j}$ and its discriminative value $t_{j}$ :

$$
\hat{\delta_{j}}=w_{j}-w_{j+1}-t_{j}=\delta_{j}-t_{j}
$$

for $j \in \llbracket m-1 \rrbracket$. Equation (25) ensures that $\hat{\delta_{j}} \geq 0$. Consider the difference of score between two alternatives $y$ and $x$ :

$$
s(y ; w)-s(x ; w)=\sum_{j=1}^{m-1} \hat{\delta}_{j}\left(V_{j}^{y}-V_{j}^{x}\right)+\sum_{j=1}^{m-1} t_{j}\left(V_{j}^{y}-V_{j}^{x}\right)
$$

We now consider the maximum $s(y ; w)-s(x ; w)$ when picking a scoring rule whose scoring vector $w$ belongs to $W^{D, t}$ (weakly decreasing and with discriminative values). Note that, the second addendum on the right-hand side of Equation (38) can be viewed as constant.

$$
\begin{array}{r}
\max _{w \in W^{D, t}} s(y ; w)-s(x ; w)=\max \left\{\sum_{j=1}^{m-1} \delta_{j}\left(V_{j}^{y}-V_{j}^{x}\right) \mid 0 \leq \delta_{j} \leq 1 \wedge \sum_{1}^{m-1} \delta_{j}=1 \wedge \delta_{j} \geq t_{j} \cdot \forall j \in \llbracket m-1 \rrbracket\right\} \\
=\sum_{j=1}^{m-1} t_{j}\left(V_{j}^{y}-V_{j}^{x}\right)+\max \left\{\sum_{j=1}^{m-1} \hat{\delta_{j}}\left(V_{j}^{y}-V_{j}^{x}\right) \mid 0 \leq \hat{\delta}_{j} \leq 1 \wedge \sum_{1}^{m-1} \hat{\delta_{j}}=1-\sum_{j=1}^{m-1} t_{j} \cdot \forall j \in \llbracket m-1 \rrbracket\right\} \\
=\sum_{j=1}^{m-1} t_{j}\left(V_{j}^{y}-V_{j}^{x}\right)+\left(1-\sum_{j=1}^{m-1} t_{j}\right) \max _{j \in \llbracket m-1 \rrbracket}\left(V_{j}^{y}-V_{j}^{x}\right) .
\end{array}
$$

Proposition 8 Let $p$ be the profile.

- When an alternative $x \in A$ is ranked first at least $\frac{2}{3} n$ times, then MMR-Dec $(p)=\{x\}$.

In other words, if $x$ is such that $v_{1}^{x}>\frac{2}{3} n$ then $S^{*}\left(W^{D}\right)=\{x\}$

- When an alternative $x \in A$ is ranked first at least $\frac{2 m-2}{3 m-2} n$ times, then MMR-Con $(p)=\{x\}$.

In other words, if $x$ is such that $v_{1}^{x}>\frac{2 m-2}{3 m-2} n$ then $S^{*}\left(W^{C}\right)=\{x\}$.

Proof 1. Decreasing weights $W^{D}$ : Consider a profile $p$ in which $x$ is such that $v^{x}=(a, 0, \ldots, 0, n-a)$ and $v^{y}=(n-a, a, 0, \ldots, 0)$ and $a>\frac{n}{2}$ ( $x$ is ranked first at least $a$ times and is last all other times; $y$ is first $n-a$ times and second $a$ times $)$. Then $V^{x}=(a, \ldots, a)$ and $V^{y}=(n-a, n, \ldots, n)$. According to Lemma 1 , $\operatorname{PMR}\left(x, y ; W^{D}\right)=V_{2}^{y}-V_{2}^{x}=n-a$ and $\operatorname{PMR}\left(y, x ; W^{D}\right)=V_{1}^{x}-V_{1}^{y}=2 a-n$. It can be shown that in this profile $\operatorname{MR}\left(x ; W^{D}\right)=\operatorname{PMR}\left(x, y ; W^{D}\right)$ and $\operatorname{MR}\left(y ; W^{D}\right)=\operatorname{PMR}\left(y, x ; W^{D}\right)$ since every alternative other than $x$ and $y$ is (weakly) dominated. Therefore $x$ is a winner if

$$
\operatorname{MR}\left(x ; W^{D}\right)<\operatorname{MR}\left(y ; W^{D}\right) \Longleftrightarrow n-a<2 a-n \Longleftrightarrow a>\frac{2}{3} n .
$$

The last step is to show that the profile $p$ is the most challenging situation to alternative $x$ : in any profile $p^{\prime}$ in which $x$ is ranked first at least $a$ times, $x$ will be at least as well ranked as in $p$, and any challenger will be at most ranked as well as $y$ in $p$. Indeed monotonicity guarantees us that the max regret of $x$ will be minimum. 
2. Convex weights $W^{C}$ : Consider a profile $p$ in which $x$ is such that $v^{x}=(a, 0, \ldots, 0, n-a)$ and $v^{y}=(n-$ $a, a, 0, \ldots, 0)$ and $a>\frac{n}{2}$ ( $x$ is ranked first at least $a$ times and is last all other times; $y$ is first $n-a$ times and second $a$ times). The cumulative ranks of $x$ and $y$ are $V^{x}=(a, \ldots, a)$ and $V^{y}=(n-a, n, \ldots, n)$; the double cumulative ranks $\mathcal{V}^{x}$ and $\mathcal{V}^{y}$ are such that that $\mathcal{V}_{j}^{x}=j a$ and $\mathcal{V}_{j}^{y}=j n-a$, with $j \in \llbracket m-1 \rrbracket$. We impose that the max regret of $x$ is lower than that of $y$, and then use Lemma 1:

$$
\begin{aligned}
& \operatorname{MR}\left(x ; W^{C}\right)<\operatorname{MR}\left(y ; W^{C}\right) \Longleftrightarrow \operatorname{PMR}\left(x, y ; W^{C}\right)<\operatorname{PMR}\left(y, x ; W^{C}\right) \\
& \Longleftrightarrow \max _{j \in \llbracket m-1 \rrbracket}\left\{\frac{\mathcal{V}_{j}^{y}-\mathcal{V}_{j}^{x}}{j}\right\}<\max _{j \in \llbracket m-1 \rrbracket}\left\{\frac{\mathcal{V}_{j}^{x}-\mathcal{V}_{j}^{y}}{j}\right\} \\
& \Longleftrightarrow \underbrace{n-\frac{a}{m-1}}_{\frac{\mathcal{\nu}_{m-1}^{y}}{m-1}}-\underbrace{a}_{\frac{\mathcal{V}_{m-1}^{x}}{m-1}}<\underbrace{a}_{\frac{\mathcal{V}_{1}^{x}}{1}}-\underbrace{(n-a)}_{\frac{\mathcal{V}_{1}^{y}}{1}} \Longleftrightarrow a>\frac{2(m-1)}{3 m-2} n .
\end{aligned}
$$

Notice that alternative $x$ is the "worst ranked" among all possible rank distribution satisfying the above condition and $y$ is ranked as well as possible (since we filled up the profile in the most disadvantageous way to $x$ ). Now to conclude the proof, as in the previous case, we make use of monotonicity to prove the argument that in any other profile where alternative $x$ satisfies the above condition on the number of first positions, $x$ will achieve minimax regret.

Theorem 3 The social choice functions MMR-Dec and MMR-Con satisfy anonymity, neutrality, unanimity, monotonicity, IRDA, homogeneity and independence from symmetric profiles.

Proof Neutrality and anonymity are trivial to check. Proof sketches for homogeneity and independence from symmetric profiles were given in the main text.

For unanimity, we need to show that if all voters place an alternative $x$ in first position, then $x$ will have a max regret value of zero and therefore $x$ will be declared the winner. $x$ has rank distribution $v^{x}=(n, 0, \ldots, 0)$ and cumulative standings $V^{x}=(n, \ldots, n)$. All other alternatives $y \neq x$ have 0 in the first component of their cumulative ranks, $V_{1}^{y}=0$, and a value less than $n$ in the other components. Using Theorem $1, \operatorname{MR}\left(y ; W^{D}\right)=n$, for any $y$, while $\operatorname{MR}\left(x ; W^{D}\right)=0$; therefore $x$ is the only winner. The proof is similar for $W^{C}$.

We now prove monotonicity. Indeed, assume that, starting from a profile $p$, we modify the ranking associated with one of the voters so that an alternative $x$ moves from some position $i_{2}$ to some position $i_{1}<i_{2}$ and call the resulting profile $p^{\prime}$. Then the rank distribution of the new profile is such that $v_{i_{1}}^{x}\left[p^{\prime}\right]=v_{i_{1}}^{x}[p]+1$ and $v_{i_{2}}^{x}\left[p^{\prime}\right]=v_{i_{2}}^{x}[p]-1$. It follows that the cumulative rank distribution $V^{x}\left[p^{\prime}\right]$ of alternative $x$ in the new profile $p^{\prime}$ is such that

$$
V_{j}^{x}\left[p^{\prime}\right]= \begin{cases}V_{j}^{x}[p] & j=1, \ldots, i_{1}-1 \\ V_{j}^{x}[p]+1 & j=i_{1}, \ldots, i_{2}-1 \\ V_{j}^{x}[p] & j=i_{2}, \ldots, m\end{cases}
$$

We therefore have $V^{x}\left[p^{\prime}\right] \succeq V^{x}[p]$. We also have $V^{y}\left[p^{\prime}\right] \preceq V^{y}[p]$ for any $y \neq x$ since $y$ can either have the same ranks as in $p^{\prime}$ or may have lose a position.

Now, let $J=\left\{j \in \llbracket m-1 \rrbracket \mid x \in \arg \max _{z} V_{j}^{z}\left[p^{\prime}\right]\right\}$, that is the set of positions for which $x$ has maximum cumulative rank in $p^{\prime}$. We have (using Theorem 1)

$$
\begin{gathered}
\operatorname{MR}_{p^{\prime}}\left(x ; W^{D}\right)=\max _{j=1}^{m-1}\left\{V_{j}^{*}\left[p^{\prime}\right]-V_{j}^{x}\left[p^{\prime}\right]\right\}=\max _{j \notin J}\left\{\max _{y \in A-\{x\}}\left\{V_{j}^{y}\left[p^{\prime}\right]\right\}-V_{j}^{x}\left[p^{\prime}\right]\right\} \leq \\
\leq \max _{j \notin J}\left\{\max _{y \in A-\{x\}}\left\{V_{j}^{y}[p]\right\}-V_{j}^{x}[p]\right\}=\max _{j=1}^{m-1}\left\{V_{j}^{*}[p]-V_{j}^{x}[p]\right\}=\operatorname{MR}_{p}\left(x ; W^{D}\right) .
\end{gathered}
$$

and this means regret of $x$ in $p^{\prime}$ cannot be higher than the regret of $x$ in $p$. Assume $y$ is any alternative that is not a winner in $p$, meaning that $\operatorname{MR}_{p}\left(x ; W^{D}\right)<\operatorname{MR}_{p}\left(y ; W^{D}\right)$. It follows

$$
\operatorname{MR}_{p^{\prime}}\left(x ; W^{D}\right) \leq \operatorname{MR}_{p}(x ; W)<\operatorname{MR}_{p}\left(y ; W^{D}\right) \leq \operatorname{MR}_{p^{\prime}}\left(y ; W^{D}\right) .
$$

Therefore, if $x$ is a winner in $p$, it has to continue to have minimax regret, and therefore to be a winner in $p^{\prime}$; we conclude that the rule is monotone in $W^{D}$. Analogous reasoning can be made for $W^{C}$.

To prove IRDA note that, according to Equation 22, the max regret of an alternative depends only on its rank distribution, and on the values $V_{1}^{*}, \ldots, V_{m-1}^{*}$. But, for any $j, V_{j}^{*}=\max _{y} V_{j}^{y}$ and the associated alternatives in $\arg \max _{y} V_{j}^{y}$ have maximal cumulative rank in the $j$-th position, meaning that are either undominated or dominated by another alternative that is also in $\arg \max _{y} V_{j}^{y}$ (said in another way, for each $j$, there is at least 1 undominated alternative in $\arg \max _{y} V_{j}^{y}$ ).

Thus any change in the rank of dominated alternatives does not change the values $V_{1}^{*}, \ldots, V_{m-1}^{*}$, when moving from $p$ to $p^{\prime}$. This means that by changing the ranks of dominated alternatives will not change $\operatorname{MR}\left(x ; W^{D}\right)$, and therefore the winners according to MMR will be the same, and therefore MMR is IRDA. 